\title{
Second Order Sliding Mode Observers for the ADDSAFE Actuator Benchmark Problem ${ }^{12}$
}

\author{
Halim Alwi ${ }^{\mathrm{a}}$, Christopher Edwards ${ }^{\mathrm{a}}$ \\ ${ }^{a}$ Structures and Dynamics Group, \\ College of Engineering, Mathematics and Physical Sciences, University of Exeter, Exeter, EX4 4QF, UK. \\ (e-mails: h.alwi@exeter.ac.uk, C.Edwards@exeter.ac.uk)
}

\begin{abstract}
This paper presents the evaluation process and results associated with two different fault detection and diagnosis (FDD) schemes applied to two different aircraft actuator fault benchmark problems. Although the schemes are different and bespoke for the problem being addressed, both are based on the concept of a second order sliding mode. Furthermore both designs are considered as 'local' in the sense that a localized actuator model is used together with local sensor measurements. The schemes do not involve the global aircraft equations of motion, and therefore have low order. The first FDD scheme is associated with the detection of oscillatory failure cases (OFC), while the second scheme is aimed at the detection of actuator jams/runaways. For the OFC benchmark problem, the idea is to estimate the OFC using a mathematical model of the actuator in which the rod speed is estimated using an adaptive second order exact differentiator. For the jam/runaway actuator benchmark problem, a more classical sliding mode observer based FDD scheme is considered in which the fault reconstruction is obtained from the equivalent output error injection signals associated with a second order sliding mode structure. The results presented in this paper summarize the design process from tuning, testing and finally industrial evaluation as part of the ADDSAFE project.
\end{abstract}

Keywords: Sliding mode observer, Fault detection and diagnosis.

\section{Introduction}

The last decade has seen many theoretical advances in sliding mode methods for Fault tolerant control (FTC) and Fault detection and isolation (FDI), and it continues to be an active area of research. The reasons for this increasing focus of attention are due to the inherent robustness characteristics of sliding modes to certain types of uncertainty ('matched uncertainty' $[1,2,3]$ ) and the ability of sliding mode observers to reconstruct unknown unmeasured signals (see for example $[4,1,5,6]$ ). The potential of using sliding mode schemes for practical and industrial applications has also to be explored. One good example is the work demonstrated in the GARTEUR FM-AG16 programme (see for example [7] for an overview of the work in this project) which studied state of the art FTC schemes applied to a realistic and challenging problem of controlling an aircraft in the event of unanticipated actuator faults or failures. Another recent project:- Advanced Fault Diagnosis for Sustainable Flight Guidance and Control (ADDSAFE), represents one of the most intensive efforts to study state of the art fault detection and diagnosis (FDD) methods applied to realistic engineering problems arising in aircraft. Within this project, a number of sliding mode design ideas for FDD have been proposed, rigorously tested and evaluated subject to industrial constraints. This paper presents the evolution of two second order sliding mode observer schemes applied to two challenging and realistic FDD benchmark problems.

One of the perceived drawbacks of sliding mode schemes for real engineering system implementations is in dealing with the discontinuities which arise from using the signum function in the injection

\footnotetext{
${ }^{1}$ This work is supported by the EU (FP7-233815) grant: ADDSAFE (Advanced Fault Diagnosis for Sustainable Flight Guidance and Control).

${ }^{2} \mathrm{~A}$ preliminary version of some of the results in this paper were presented at the IFAC SAFEPROCESS workshop, Mexico City, Mexico, September 2012.
} 
signals [3]. There has been extensive research over the years to overcome this problem ranging from simple so-called pseudo-sliding approaches through smoothing (see for example [2]), to more sophisticated higher order sliding mode concepts (see for example $[8,5,9,10]$ ). So-called second order sliding mode schemes are considered in this paper as a basis for the FDD schemes for a number of reasons. The main reason is that in 2 nd order sliding mode schemes, an ideal sliding motion can still be achieved with less chattering, without resorting to using approximations of the signum function (see for example $[8,5,9,10]$ ) or the need to filter discontinuous signals to extract the equivalent injections (which contain the fault information). Although it may be argued that chattering is not really a significant issue in FDD applications, filtering the output error injection signal to create the equivalent injection signal is not always the best option as it creates phase delays and crucially slows down the detection time. This is not ideal since often industrial specifications require certain types of fault to be detected very quickly. An approximate signum function can be used to replace the true discontinuity but this may in certain circumstances degrade the level of reconstruction performance, as sliding is only maintained in a boundary around the sliding surface, but not on the surface itself.

The results presented here concern two different FDD detection problems and use two bespoke sliding mode based FDD strategies. However both proposed designs are based on the same underlying concept: namely a second order sliding mode strategy known as 'super-twisting' [10]. Both designs are considered as 'local' approaches as they only involve a local actuator model and associated (local) measurements, and do not involve the 'global' aircraft equations of motion. The first ADDSAFE benchmark problem pertains to the detection of oscillatory failure cases (OFC) in the actuator servo loop. The idea is to detect unwanted un-commanded oscillations due to faults in the servo loop system. If undetected these oscillations could cause excessive loads on the structure of the aircraft. The idea is to provide robust prompt detection of any unwanted oscillations thus obviating the need for additional structural strengthening of the aircraft. This reduction of weight at the design stage ultimately translates to a reduction in fuel burn, therefore reducing fuel consumption and costs in the longer term. The idea is to use a second order exact differentiator scheme with adaptive gains, as proposed in [11], in order to estimate the actuator rod speed. This estimation of the rod speed is used as part of the estimation of any OFCs that may be present in the servo loop, by mathematical manipulation of the actual actuator model. The scheme allows OFCs to be reconstructed, which together with oscillation counting (for example using the existing scheme described in [12]), allows OFCs to be detected. This is particularly useful for low and medium frequency OFCs. However, for high frequency OFCs which requires prompt detection, the adaptive scheme will be used to provide faster detection than can be obtained by oscillation counting. The idea is that for low frequency and low amplitude OFCs, only small gains are required in the super-twist scheme. However, in high frequency and high amplitude cases, larger gains are required in order to maintain a sliding motion and hence the quality of the reconstruction. Thus any increase in the adaptive gain above a certain threshold will indicate the presence of high frequency and high amplitude OFCs, and detection can be provided much earlier than with the existing oscillation counting schemes.

The second ADDSAFE FDD benchmark problem considered here is concerned with actuator jams and runaways. The design uses a more classical sliding mode observer based FDD scheme which provides a reconstruction of the fault. In the benchmark model, the faults are represented as an additive fault on the output of the actuator servo loop. The reconstruction of the localized actuator output fault is achieved by transforming the output fault problem into a standard input fault reconstruction problem. This is done by augmenting the local nonlinear actuator model with a filtered version of the measured output thus creating a second order system about which the observer design is based. The fault reconstructions are then provided by the output error injection signal associated with the second order sliding mode scheme.

The results presented in this paper summarize the design process for the two proposed second order sliding mode FDD schemes, from tuning, testing through to final verification and validation using Monte-Carlo testing as part of the industrial evaluation process. The results presented in this paper have been obtained using the full nonlinear ADDSAFE benchmark aircraft model provided by AIRBUS, which has been incorporated into a Functional Engineering Simulator (FES) for an 
automated sweep of the entire flight envelope with varying uncertainty levels.

\section{ADDSAFE}

The Advanced Fault Diagnosis for Sustainable Flight Guidance and Control (ADDSAFE) project aims to address the challenges associated with future 'sustainable' (cleaner, quieter smarter and more affordable) aircraft $[13,14]$. The aim of ADDSAFE is to demonstrate the applicability of advanced FDD methods to support the development of sustainable aircraft. It poses challenges to improve the FDD schemes which support new 'green technologies' allowing optimization of the aircraft structural design, improving aircraft performance and reducing the environmental footprint $[15,13,14]$.

The FDD challenges considered in the ADDSAFE project consist mainly of actuator and sensor malfunctions and include the flight parameter management system, abnormal aircraft behaviour and servo-loop actuator/sensor faults $[13,14]$. The results presented in this paper concentrate on two localized servo-loop actuator faults, specifically: an oscillatory fault case and an actuator jam and runaway.

\subsection{ADDSAFE benchmark model}

The ADDSAFE nonlinear aircraft benchmark model, provided by AIRBUS $[16,13,14,17]$ represents a high fidelity model of a rigid body twin engine, civil commercial aircraft, with highly detailed nonlinear actuator and sensor models, together with a complex aerodynamic database. The model (which includes pilot side stick and pedals inputs, and a flight control law to provide closed-loop nonlinear aircraft autopilot capabilities) is highly representative of the actual aircraft flight physics with realistic handling qualities [16].

\section{2. $A D D S A F E$ FES}

This model has been incorporated within a functional engineering simulator (FES) which is a dedicated validation tool developed by DEIMOS $[18,14]$. The ADDSAFE-FES is a simulator which runs under a MATLAB/SIMULINK and XML modelling and simulation environment. It is intended for simulation and performance assessment of the FDD algorithms developed within ADDSAFE and provides the necessary 'infrastructure' to run various simulation tests (including parametric runs and intensive Monte-Carlo campaigns to cover a wide range of the flight envelope, conditions and manoeuvres). It also provides facilities for the post processing of simulation data and performance evaluation $[18,14]$.

\begin{tabular}{|l|c|c|}
\hline Features & $\begin{array}{c}\text { simulation and } \\
\text { verification FES }\end{array}$ & $\begin{array}{c}\text { Industrial Benchmarking and } \\
\text { Validation FES }\end{array}$ \\
\hline FDD benchmark model & $\checkmark$ & $\checkmark$ \\
pre-defined scenarios & $\checkmark$ & $\checkmark$ \\
visualization of simulation outputs & $\checkmark$ & $\checkmark$ \\
failure detection metrics & $\checkmark$ & $\checkmark$ \\
Monte Carlo simulation & $\times$ & $\checkmark$ \\
Simulation campaign mode & $\checkmark$ & $\checkmark$ \\
Automatic batch simulations & $\checkmark$ & $\checkmark$ \\
\hline
\end{tabular}

Table 1: simplified vs. Industrial FES comparison [18]

Two versions of the ADDSAFE-FES have been developed; the first is the simulation and verification FES (simplified version) which is intended to provide simulation and verification tools to facilitate tuning of the FDD methods. The second is the industrial benchmarking and validation FES used to validate and evaluate the selected FDD designs. The differences between the two versions of the ADDSAFE-FES are given in Table 1 [18]. The most significant difference is the Monte Carlo approach which is only available in the industrial FES. 


\subsubsection{Simulation and Verification FES (Parametric FES)}

The simplified simulation and verification FES uses parametric sweeps of the flight conditions with fixed grid parameter dispersions instead of random Monte Carlo dispersions [18, 14]. The results are obtained for a particular flight manoeuvre (depending on the fault type $[19,14]$ ) with variations in the operating conditions (altitude $(h)$, speed $\left(V_{c a s}\right)$, mass $(m)$, center of gravity $\left(X_{c g}\right)$ ) and uncertainty in the aerodynamic coefficients of the roll pitch and yaw moments $\left(C_{l}, C_{m}, C_{n}\right)$ and the coefficients of forces in the $X, Y, Z$ axes $\left(C_{X}, C_{Y}, C_{Z}\right)$, and imprecise knowledge of altitude $h$, speed $V_{\text {cas }}$, mass $m$, and center of gravity $X_{c g}$. The simulation campaign sweeps the entire flight envelope using a combination of grid parameters given in Table $2(3 \times 3 \times 4 \times 3=108$ possible combinations). For each of these combinations, three additional sequences based on the minimum, nominal and maximum levels of uncertainties in the aerodynamic coefficients and sensor measurements have also been tested. This results in a total of 324 simulation runs for each type of fault (including fault free runs).

\begin{tabular}{|c|cccc|}
\hline grid & dispersion & & & \\
param & 1 & 2 & 3 & 4 \\
\hline$m(\times 1000 K g)$ & 120 & 180 & 233 & \\
$X_{c g}(\%)$ & 17 & 30 & 41 & \\
$h(\times 1000 f t)$ & 8 & 18 & 28 & 38 \\
$V_{\text {cas }}(k t s)$ & 160 & 220 & 300 & \\
\hline
\end{tabular}

\begin{tabular}{|c|ccc|}
\hline $\begin{array}{c}\text { sequence } \\
\text { parameters }\end{array}$ & dispersion & & \\
min & nom & $\max$ \\
\hline$\delta C_{X}, \delta C_{Y}, \delta C_{Z}(\%)$ & -5 & 0 & 5 \\
$\delta C_{l}, \delta C_{m}, \delta C_{n}(\%)$ & -5 & 0 & 5 \\
$\delta m(\%)$ & -10 & 0 & -10 \\
$\delta X_{c g}(\%)$ & -10 & 0 & -10 \\
$\delta h(\%)$ & -10 & 0 & -10 \\
$\delta V_{\text {cas }}(\%)$ & -10 & 0 & -10 \\
\hline
\end{tabular}

Table 2: Grid and sequence for parameter dispersion [18]

\subsubsection{Industrial Benchmarking and Validation FES (Industrial FES)}

For the industrial FES, a similar setup was considered with variations in operating conditions, perturbations and uncertainty. Now however, these variations are associated with a Monte-Carlo setup to provide a random sweep of the entire flight envelope to assess the robustness and performance of the proposed FDD designs. In total 2200 runs have been conducted which are composed of 200 runs for six different fault free manoeuvres $(6 \times 200=1200$ runs $)$, and 1000 runs for the faulty case. This is intended to represent the industrial evaluation for the ADDSAFE benchmark problems. Once the Monte Carlo simulation campaign is invoked, no further modification of the designs has been made.

Remark: Note that due to the large amount of data generated during the simulations, only the most significant plots will be presented in this paper. All the fault types considered are from the scenarios associated with the ADDSAFE benchmark problem as defined in [19, 14]. Further details about the FES can be found in [14, 18].

\subsection{Design, testing, evaluation and validation process}

In this paper different sets of results will be presented (see Table 3) to demonstrate the different stages of the design from initial tuning (using the ADDSAFE model) to testing (in the simplified FES) and finally the industrial benchmarking evaluation and verification process ${ }^{3}$ (using the industrial FES). This highlights the evolution of the designs and the steps which have been taken in the process of migrating the design from an 'academic' environment, into a more practical and rigorous implementation testing environment.

The single run tests provide a preliminary first level evaluation with no uncertainties or variations in the flight conditions. This single run test is used as a 'controlled case' with known faults, to check that the observer is working as expected. The second level of tests are conducted on the Simulation and Verification FES with grid parametric dispersion. This is used for advanced tuning to ensure that

\footnotetext{
${ }^{3}$ supervised by the industrial partners within the ADDSAFE consortium represented by AIRBUS and DEIMOS.
} 


\begin{tabular}{|l|c|c|c|}
\hline Fault type & single run & $\begin{array}{c}\text { Simulation and } \\
\text { Verification FES }\end{array}$ & $\begin{array}{c}\text { Industrial Benchmarking and } \\
\text { Validation FES }\end{array}$ \\
\hline left elevator OFC & $\checkmark$ & $\checkmark$ & $\checkmark$ \\
right elevator jam \& runaway & $\checkmark$ & $\checkmark$ & $\checkmark$ \\
\hline
\end{tabular}

Table 3: Level of tests conducted

the design provides good performance over a wide range of the flight envelope in preparation for the industrial benchmarking and validation. The final tests are conducted on the Industrial Benchmarking and Validation FES with full Monte-Carlo dispersion to cover the flight envelope.

\section{OFC Benchmark Problem}

An OFC is a type of failure in the Electrical Flight Control System (EFCS) which leads to additional loads on the aircraft structure that must be taken into account in terms of structural design [12]. These loads are a function of the amplitude and duration of the oscillation, and therefore if an OFC can be detected quickly at a low level, less load is imposed on the structure and the aircraft remains within the original load envelope design. Any improvement in the performance of the FDD scheme used to detect OFCs will allow better optimization of the aircraft structural design, which translates to weight savings, less fuel burn and a lower environmental footprint. The earliest published work on OFC detection can be found in [20] which examines the effect of Electronic Flight Control Systems (EFCS) failures on the structural load on aircraft. In contrast, [21] provides an overview of the a state of the art OFC detection schemes currently in service on the AIRBUS A380. The recent work described in $[22,23]$ is an example of work on OFC detection outside of ADDSAFE, and proposes a residual generation approaches using a nonlinear observer design with an oscillation counting mechanism similar to the one in [21] to detect the presence of OFCs. Other recent work on OFC detection from within ADDSAFE can be found in [24, 25, 26, 27, 28]. In particular [26] applies higher order sliding mode ideas as part of a hybrid scheme involving an online iteration based algebraic equation solver.

\subsection{Modeling of an hydraulic actuator with OFC}

In [21], a nominal fault free hydraulic actuator model (obtained from first principles) is given by

$$
\dot{x}(t)=V_{0}(t)\left(\frac{\Delta_{p_{t o t}}(t)}{\Delta_{p_{r e f}}}\right)^{\frac{1}{2}}
$$

where

$$
V_{0}(t)=K_{c} i(t)=K_{c} K(u(t)-x(t))
$$

In equation (2), $x(t)$ is the hydraulic actuator rod position, $u(t)$ is the commanded rod position (from the flight control computer (FCC),$i(t)$ is the electrical current supplied to the actuator, while the gain $K_{c}$ represents a model of the servovalve and $K$ is the (fixed) servo control gain. The fixed constant $\Delta_{p_{\text {ref }}}$ represents the differential pressure corresponding to the maximum rod speed. The quantity $\Delta_{p_{t o t}}(t)$ is the total pressure consisting of supply, aerodynamic and damping pressure given by

$$
\begin{aligned}
\Delta_{p_{\text {tot }}}(t) & =\Delta_{p}(t)-\left(\Delta_{p_{\text {aero }}}(t)+\Delta_{p_{\text {damping }}}(t)\right) \\
& =\Delta_{p}(t)-\left(\frac{\operatorname{sign}(i(t)) F_{\text {aero }}(t)+F_{\text {damping }}(t)}{S}\right)
\end{aligned}
$$

where $\Delta_{p}(t)$ is the actual hydraulic pressure delivered to the actuator, $F_{\text {aero }}(t)$ is the aerodynamic force and $S$ is the piston surface area. The damping force $F_{\text {damping }}(t)$ can be written as

$$
F_{\text {damping }}(t)=K_{d}(t) \dot{x}^{2}
$$


where $K_{d}(t)$ is the adjacent actuator damping coefficient (in case of two actuators per control surface). Using (4) and (3) after algebraic manipulation, equation (1) can be written in explicit form as:

$$
\dot{x}(t)=V_{0}(t)\left(\frac{\Delta_{p}(t)-\operatorname{sign}(i(t)) \frac{F_{a e r o}(t)}{S}}{\Delta_{p_{\text {ref }}}+\frac{K_{d}(t)}{S} V_{0}^{2}(t)}\right)^{\frac{1}{2}}
$$

In the equation above the variables $\Delta_{p}(t), F_{\text {aero }}(t)$ and $K_{d}(t)$ evolve based on changes in the operational conditions, and link to the other components of the ADDSAFE benchmark model via these quantities.

An OFC is usually caused by faults in digital components in the actuator control loop between the FCC and the control surface, and results in unwanted sinusoidal signals. In the benchmark, the amplitude and frequency of the OFCs are assumed to be uniformly distributed in the range $1-10 \mathrm{~Hz}$ $[19,21]$. (Due to the low pass characteristics of the actuator, the OFC has no effect on the control surface beyond $10 \mathrm{~Hz}[19,21])$.

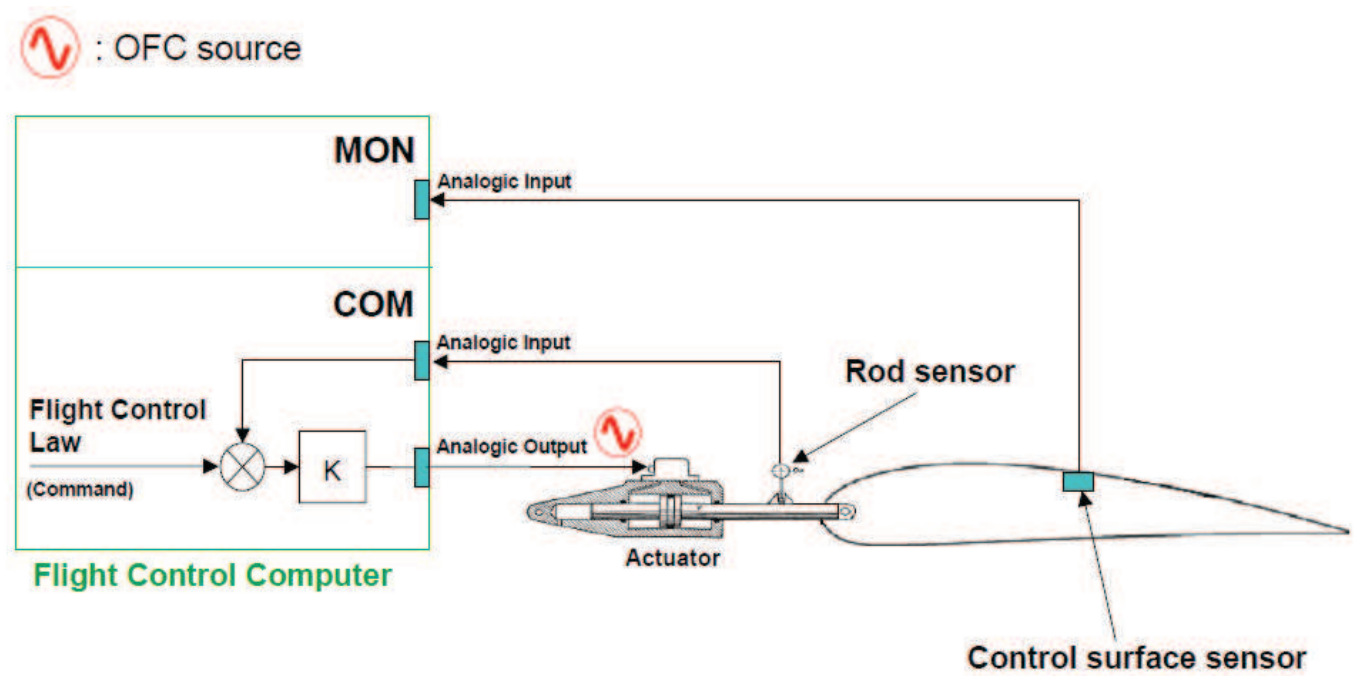

Figure 1: Source of OFC in the servo control loop [21]

In the ADDSAFE benchmark (Figure 1), the OFC affects the computed/desired rod speed denoted by $V_{c}(t)[19,21]$. Two types of OFC are considered - so-called liquid OFCs and solid OFCs [21]. The hydraulic actuator with an OFC fault is modelled as

$$
\dot{x}(t)=V_{c}(t)\left(\frac{\Delta_{p}(t)-\operatorname{sign}(i(t)) \frac{F_{a e r o}(t)}{S}}{\Delta_{p_{r e f}}+\frac{K_{d}(t)}{S} V_{c}^{2}(t)}\right)^{\frac{1}{2}}
$$

where $V_{c}(t)$ takes one of the three possible forms:

$$
V_{c}(t)= \begin{cases}V_{0}(t) & \text { nominal } \\ V_{0}(t)+K_{c} f_{l i q}(t) & \text { liquid OFC } \\ K_{c} f_{\text {sol }}(t) \text { i.e. } V_{0}(t)=0 & \text { solid OFC }\end{cases}
$$

and $V_{0}(t)$ and $K_{c}$ are defined in (2). In (7), $f_{l i q}(t)$ and $f_{\text {sol }}(t)$ represent the fault signals which induce liquid and solid OFC faults respectively. As shown in (7) a liquid OFC behaves as a corruption to the desired position from the FCC and essentially the control surface tracks an incorrect demand signal. For the case of solid OFCs, the demanded surface position is totally replaced by the OFC signal and the control surface is totally 'disconnected' from the FCC and does not respond to any desired rod position commands. Any attempt to damp the oscillation (by the FCC) does not have any impact as the control surface is 'disconnected' from any demand signal [21].

Remark: Note that in the ADDSAFE actuator model, the source of the OFC is in the analogic output of the servo loop [21]. The signals $f_{l i q}$ and $f_{\text {sol }}$ in (7) do not directly refer to the fault oscillation at the actuator rod itself, but rather correspond to the fault signal injected in the electrical current 
supplied to the actuator and therefore the actual unit of the source of the fault is milliamperes $(\mathrm{mA})$. Note that even though the injected fault is in the electrical current supply $(\mathrm{mA})$, the effect of the oscillatory fault propagates through the servo loop and can be seen on the rod position signal ( $\mathrm{mm}$ ) and the control surface deflection (deg) [21].

\subsection{OFC Estimation}

In this section, an adaptive second order sliding mode observer algorithm will be used to estimate actuator oscillatory faults. The idea is to reconstruct the OFC by manipulating the analytical mathematical nonlinear model of the actuator given in (6) to obtain an expression for the OFC signal. Most of the parameters used in the manipulated nonlinear equation are available or can be estimated, except for the actuator rod speed which will be supplied by a second order exact differentiator scheme.

In this section, an observer based on the ideas proposed in [29] is considered. A novel aspect of the reformulation is that the gains will be allowed to adapt. This will ensure a good estimate of rod speed even in the presence of an OFC. In fault free conditions, a 'small gain' is sufficient to ensure a good estimate of rod speed while ensuring that the effect of noise is not accentuated. For this particular application, this is very important since the observer must function in a noisy environment. However, with small gains, when high amplitude/frequency OFCs occur, sliding is broken thus compromising the estimate of the rod speed. In this case, larger gains are required to ensure a sliding motion is maintained.

Consider equation (6) as a special case of the differential equation

$$
\dot{x}(t)=g(t, x, u, f)
$$

with measured output $y(t)=x(t)$ where $f(\cdot)$ represents potential faults in the system and $u(t)$ is a known input signal. Note that even though in the benchmark model the actuator parameters $\Delta_{p}, F_{\text {aero }}$ and $K_{d}$ vary based on changes in the operational conditions, when creating the differential equation in (8) for observer design purposes, these parameters are assumed to be fixed at constant (nominal) values. Assume that the time derivative of the function on the right hand side of (8) is bounded i.e.,

$$
|\dot{g}(t, x, u, f)| \leq \delta
$$

for some constant $\delta>0$. This is realistic since physically $\dot{g}(t, x, u, f)$ represents the acceleration of the actuator rod which is bounded.

Consider an observer of the form

$$
\begin{aligned}
& \dot{z}_{1}(t)=-\kappa_{1}(t)\left|e_{1}(t)\right|^{1 / 2} \operatorname{sign}\left(e_{1}(t)\right)+z_{2}(t) \\
& \dot{z}_{2}(t)=-\kappa_{2}(t) \operatorname{sign}\left(e_{1}(t)\right)
\end{aligned}
$$

where $e_{1}(t)=z_{1}(t)-x(t)$ is the difference between $z_{1}(t)$ and the measured rod position. In (10)-(11) the scalars $\kappa_{1}(t), \kappa_{2}(t)$ are adaptive gains to be defined later. Subtracting (8) from (10) yields the error system

$$
\begin{aligned}
& \dot{e}_{1}(t)=-\kappa_{1}(t)\left|e_{1}(t)\right|^{1 / 2} \operatorname{sign}\left(e_{1}(t)\right)+z_{2}(t)-g(t, x, u, f) \\
& \dot{z}_{2}(t)=-\kappa_{2}(t) \operatorname{sign}\left(e_{1}(t)\right)
\end{aligned}
$$

Define $e_{2}(t)=z_{2}(t)-g(t, x, u, f)$, then the system in (12)-(13) can be equivalently written as

$$
\begin{aligned}
& \dot{e}_{1}(t)=-\kappa_{1}(t)\left|e_{1}(t)\right|^{1 / 2} \operatorname{sign}\left(e_{1}(t)\right)+e_{2}(t) \\
& \dot{e}_{2}(t)=-\kappa_{2}(t) \operatorname{sign}\left(e_{1}(t)\right)-\dot{g}(t, x, u, f)
\end{aligned}
$$

If a 2nd order sliding motion $[6,5,8]$ is induced, $e_{1}(t)=\dot{e}_{1}(t)=0$ in finite time and therefore from (14), $e_{1}(t)=\dot{e}_{1}(t)=0 \Rightarrow e_{2}(t)=0 \Rightarrow z_{2}(t)=g(t, x, u, f)=\dot{x}(t)$. Consequently $z_{2}(t)$ from (11) provides the estimate of rod speed $\dot{x}(t)$. Since (from the observer) both $x(t)$ and $\dot{x}(t)$ are known in 
finite time, under the three different conditions in (7), equation (6) can be rearranged to obtain an expression for the faulty signals given by

$$
\begin{aligned}
& \hat{f}_{\text {liq }}(t)=\frac{z_{2}(t) \hat{f}(t)-V_{0}(t)}{K_{c}} \\
& \hat{f}_{\text {sol }}(t)=\frac{z_{2}(t) \hat{f}(t)}{K_{c}}
\end{aligned}
$$

where

$$
\hat{f}(t)=\left(\frac{\Delta_{p_{\text {ref }}}}{\Delta_{p}(t)-\operatorname{sign}(i(t)) \frac{F_{\text {aero }}(t)}{S}-z_{2}^{2}(t) \frac{K_{d}(t)}{S}}\right)^{\frac{1}{2}}
$$

Assuming $\Delta_{p}, F_{\text {aero }}$ and $K_{d}, \Delta_{p_{\text {ref }}}, K, S$ are known or can be estimated, the quantities $\hat{f}_{\text {liq }}(t)$ and $\hat{f}_{\text {liq }}(t)$ are available in realtime.

In this paper, the gains $\kappa_{1}(t)$ and $\kappa_{2}(t)$ are chosen as:

$$
\kappa_{1}(t)=\sqrt{2 \Gamma(t)}, \kappa_{2}(t)=4 \Gamma(t)
$$

for some time varying scalar

$$
\Gamma(t)=r(t)+r_{0}
$$

where the variable $r_{0}$ is a fixed positive scalar while the varying $r(t)$ scalar (also positive), is adapted based on the law

$$
\dot{r}(t)= \begin{cases}\gamma D\left(\left|e_{1}(t)\right|^{1 / 2}\right) & \text { if } r(t) \leq r_{\max } \\ 0 & \text { otherwise }\end{cases}
$$

where $D(z): \mathbb{R} \mapsto \mathbb{R}$ is the dead-zone function

$$
D(z)= \begin{cases}0 & \text { if }|z|<\epsilon \\ z & \text { otherwise }\end{cases}
$$

and $\epsilon$ is a positive scalar. The quantity $r_{\max }$ is the largest possible value that $r(t)$ is allowed to take. The idea is to adapt the gains when $\left|e_{1}(t)\right|^{1 / 2}$ unacceptably deviates from zero when sliding is broken. The gain $r(t)$ will increase in magnitude according to (21) to force $e_{1}(t)$ back into a sliding regime. Formally it is shown in [11] that the adaptation rule (21) ensures $r(t)$ remains bounded and the error system (14)-(15) enters a small neighbourhood of the origin depending on the size of $\epsilon$. In practice, provided, as a result of the adaptation $\Gamma(t)$ becomes sufficiently large so that $\Gamma(t)>|\dot{g}(t, x, u, f)|$ over a sufficiently long finite interval $\left[t_{0}, t_{1}\right]$, then $e_{1}(t)=\dot{e}_{1}(t)=0$ in finite time.

There are three tuning parameters associated with the adaptive differentiator, namely $\gamma, \epsilon, r_{0}$. The selection of these parameters depends strongly on the system requirements and therefore requires some design iteration. The gain $r_{0}$ represents the nominal gain when adaptation is not required, whilst $\gamma$ will influence how fast the adaptive gain $r(t)$ increases. The parameter $\epsilon$ dictates the sensitivity of $r(t)$ to changes in $e_{1}$ and is set to be small. In this problem a significant concern is the effect of noise on the measurement of the $\operatorname{rod} x(t)$. The idea is to select $\epsilon$ larger than the expected noise level so that no adaptation takes place under normal fault free operations, and that adaptation is indicative of the presence of either large amplitude OFCs or OFCs of high frequency.

Remark: The approach adopted here is very bespoke to the problem formulation associated with (6)-(7). In particular the 'generic' approaches from [1] cannot be applied directly. The reason is that, whilst in (7) the faulty signals $f_{l i q}$ and $f_{\text {sol }}$ appear linearly, the overall result obtained from combining (7) with (6) yields a representation which is nonlinear with respect to both $f_{\text {liq }}$ and $f_{\text {sol }}$ : hence the need to develop a specific formulation.

Remark: With respect to gain adaptation, the approach in this paper is quite separate from the work in [30]. In [30], whilst the gains in the super-twisting algorithm do indeed change with respect to time, they do so as fixed expressions of known bounding functions for the uncertainty. Here the 
scheme adapts based on the purity of the sliding motion and does not assume any particular form for the bounding function on the uncertainty or disturbance. Using a fixed worst case upper-bound on the effect of the faults to design the differentiator gains is not ideal in this particular problem. This constant bound can be small for low frequency OFCs but must be large for high frequency or large amplitude OFCs. In the paper the adaptation mechanism is an attempt to maintain sliding in the presence of high frequency and large amplitude OFCs to ensure sliding is maintained, so that the reconstruction signal accurately estimates the sinusoidal wave forms present. It is important to maintain this level of accurate reconstruction to distinguish between an OFC and an actuator runaway for example, in which the control surface moves uncommanded, at maximum rate, to an extreme position (so-called hard-over) ${ }^{4}$. Once the gain has adapted (i.e. increased) it is not imperative to reduce it, because as soon as the fault is detected, the primary actuator is disconnected from the FCC to remove the source of the oscillation, and a backup (secondary) actuator is deployed to move the surface.

\section{3. $O F C$ : Detection logic}

The detection for the OFC type fault relies on the fault reconstruction signal and then oscillation counting is used to confirm the presence of a fault and raises the detection signal alarm. For extreme high frequency OFCs, fault detection is confirmed when the adaptive gain exceeds a preset threshold.

\subsection{Simulations}

Two levels of simulation results will be presented here: firstly 'single runs' (with no uncertainty which represent results from the preliminary first level tuning) will be shown; and secondly FES parametric runs with gridded flight envelope coverage and parametric uncertainty (results from advance tuning for industrial evaluation) will be demonstrated.

The design parameters from (20) and (21) used in the simulation are $\gamma=2 \times 10^{4}, r_{0}=50$ and $\epsilon=0.45$. To ensure a design which satisfies the restriction on computational power load associated with the flight computer, the simulations are performed using fixed time step of $0.01 \mathrm{~s}$ within the ordinary differential equation (ODE) solver. The scheme proposed above has been tested on the nonlinear high fidelity ADDSAFE benchmark model provided by AIRBUS [17, 14, 13, 16]. In the benchmark model, the actuators are represented as high fidelity nonlinear models with parameters $\Delta_{p}, F_{\text {aero }}$ and $K_{d}$ which vary based on changes in the operational conditions. For the observer design, these parameters are assumed to be fixed at constant values. The OFC is considered to appear in the analogic output of the FCC (as shown in Figure 1) used to send the electrical current signal, which provides the desired actuator position to the solenoid valve of the actuator. Here, the control surface considered is the left elevator and the requirements specify that an OFC needs to be detected within 3 cycle periods for the purpose of the ADDSAFE project.

Remark: Note that the specific values of the aircraft parameters $\left(\Delta_{p}, F_{\text {aero }}\right.$ and $\left.K_{d}, \Delta_{p_{\text {ref }}}, K, S\right)$ are not given here due to industrial confidentiality restrictions. Also note that for the same reason, some of the plots in the following figures are expressed in terms of percentage of the admissible range.

\subsection{Single run simulation results}

The scheme proposed above has been tested on the nonlinear high fidelity ADDSAFE benchmark model and the control surface considered is the left elevator. The simulations are conducted at an altitude of $30000 \mathrm{ft}$, Mach 0.64 (241kts), a weight of 200 tonnes and center of gravity at $30 \%$ of the mean aerodynamic chord (MAC). Although various OFC amplitudes and frequencies have been tested, only a representative set of results are presented here. In the simulation, the aircraft is excited by a pilot longitudinal stick doublet input and the OFC is set to occur at 10sec.

Figures 2 and 3 show the results for both liquid and solid OFCs of medium amplitude and moderate frequency. Figures 2(a) and 3(a) show the effect of OFCs on the elevator deflection and the estimated

\footnotetext{
${ }^{4}$ This scenario is discussed in detail later in the paper.
} 
actuator rod speed. Figures 2(b) and 3(b) show the gain is adapted once the threshold is exceeded (i.e. once $\left|e_{1}(t)\right|^{0.5}>\epsilon=0.45$ ). Finally Figure 2(c) and 3(c) show good reconstruction of both the liquid and solid fault signals (the reconstruction overlaps the actual signal).

\subsection{Parametric Simulation Results}

Various OFC amplitudes and frequencies under different flight conditions have been tested for the left elevator. For consistency and for comparison, all the tests on the left elevator were conducted using the same manoeuvre (a so-called angle of attack protection manoeuvre). For brevity, only the plots for left elevator liquid with low frequency and small amplitude are shown here.

\subsubsection{Elevator Liquid OFC Parametric run}

Figure 4 shows the smallest amplitude and lowest frequency OFC the scheme is required to detect within the ADDSAFE specifications. Figure 4(a) shows the variation of the states associated with the grid parameters. Figure 4(b) shows the commanded and the actual surface deflections and shows the effect of low frequency and small amplitude OFCs on the left elevator for all 324 runs. Figure 4(c) shows the output of the FDD scheme. The left plot of 4(c) shows the absolute value of the fault reconstruction signal which is used for fault detection shown in the right plot. The fault is set to occur at 20.3sec during the angle of attack protection manoeuvre. It can be seen from Figure 4(c) that the OFC is detected well before 3 cycle (26sec).

\subsubsection{Parametric FES statistics}

Tables 4 show the statistical results from the parametric runs under various amplitude and frequency OFC faults. These results show no false alarms during fault free cases and good detection times within the three OFC cycle limit required by ADDSAFE for the faulty cases. Note that due to industrial confidentiality requirements, the amplitude and frequency of the OFC faults are described in terms of 'small' or 'big' with respect to amplitude, and 'slow', 'medium' or 'fast' with respect to frequency. Also note that the maximum FDD detection time $t_{D_{f d d}} \max$ are normalized values. A value of 1 indicates that the fault is detected before 3 cycles, while any value between 0 and 1 indicates a faster than required detection time (before 3 cycles, which is highly desirable).

In addition to testing the FDI schemes during straight and level flight cruise conditions (at different altitudes and speeds), the schemes have been tested using a variety of manoeuvres designed to excite the longitudinal and lateral dynamics. The ' $\alpha$-protection' and 'nose-up' manoeuvres excite the longitudinal dynamics. In the former, via the inputs, the aircraft is forced to execute a manoeuvre which results in a large angle of attack which is sufficient to trigger the ' $\alpha$-protection' control mode within the FCS, which aims to ensure that the aircraft does not exceed a preset value of ' $\alpha_{\text {max }}$ ' even if the pilot applies full aft stick deflection. In the nose-up manoeuvre, the aircraft is forced to fly in an unusual flight condition in which the pitch angle is abnormally large. This is carried out to bring the aircraft close to the limits of normal flight. In the pitch protection manoeuvre the aircraft is forced into a regime which triggers the pitch attitude protection mode whereby the pitch is limited between preset minimum and maximum values. In the yaw angle mode the aircraft tracks and maintains a yaw angle command. Finally in the 'coordinated turn' manoeuvre, combined longitudinal and lateral inputs are applied to change heading whilst maintaining altitude and speed.

\section{Actuator Jam/runaway Benchmark Problem}

In this section, an FDD scheme for the ADDSAFE actuator jam/runaway benchmark problem is presented. Small actuator jams result in non-optimized trim conditions. These non-optimized surface deflections will cause unnecessarily higher drag, and if left undetected, can result in unnecessary fuel consumption. A runaway actuator can result in undesirable high loads on the aircraft structure and must be detected very quickly. The ADDSAFE specifications require the runaway fault to be detected before excessive loads appear on the structure of the aircraft. A robust fast FDD scheme allows a reduction in the structural reinforcement required to deal with this situation. This structural 

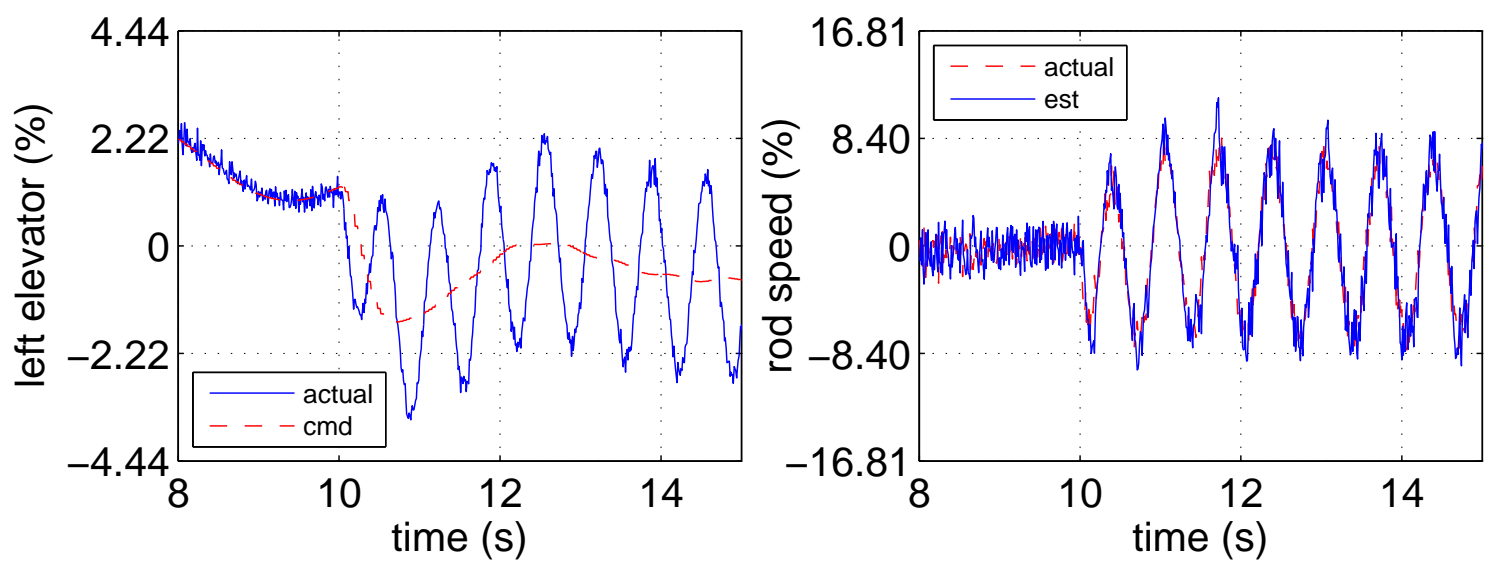

(a) control surface deflection and rod speed
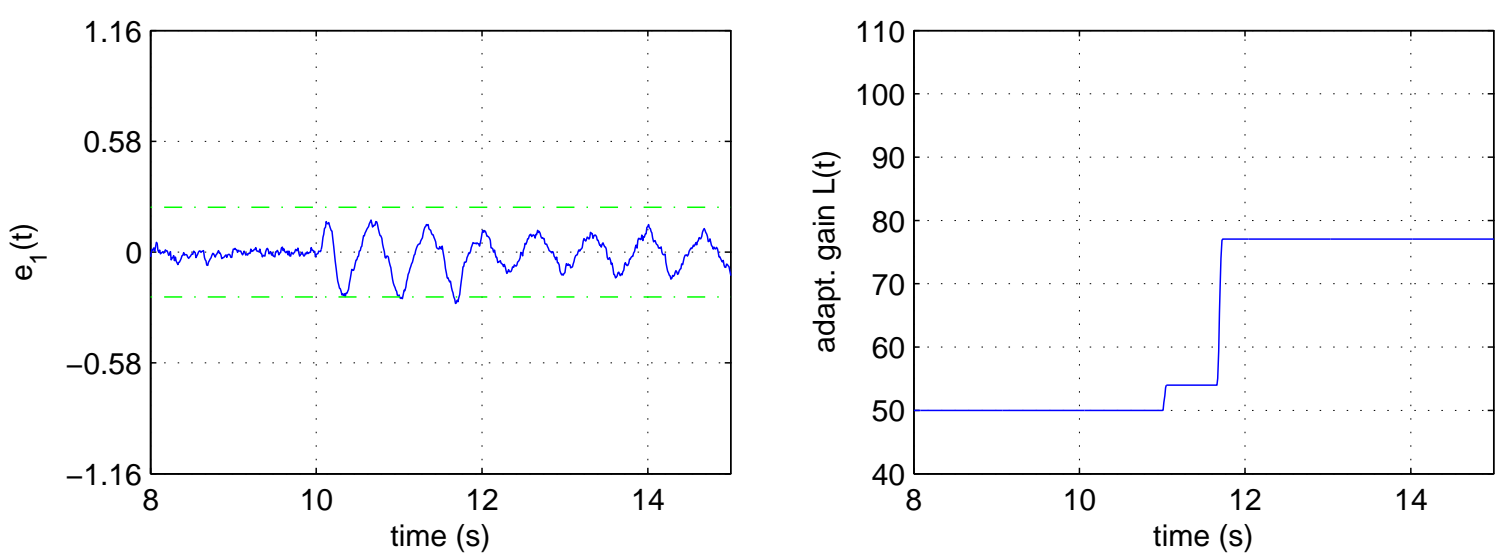

(b) observer performance

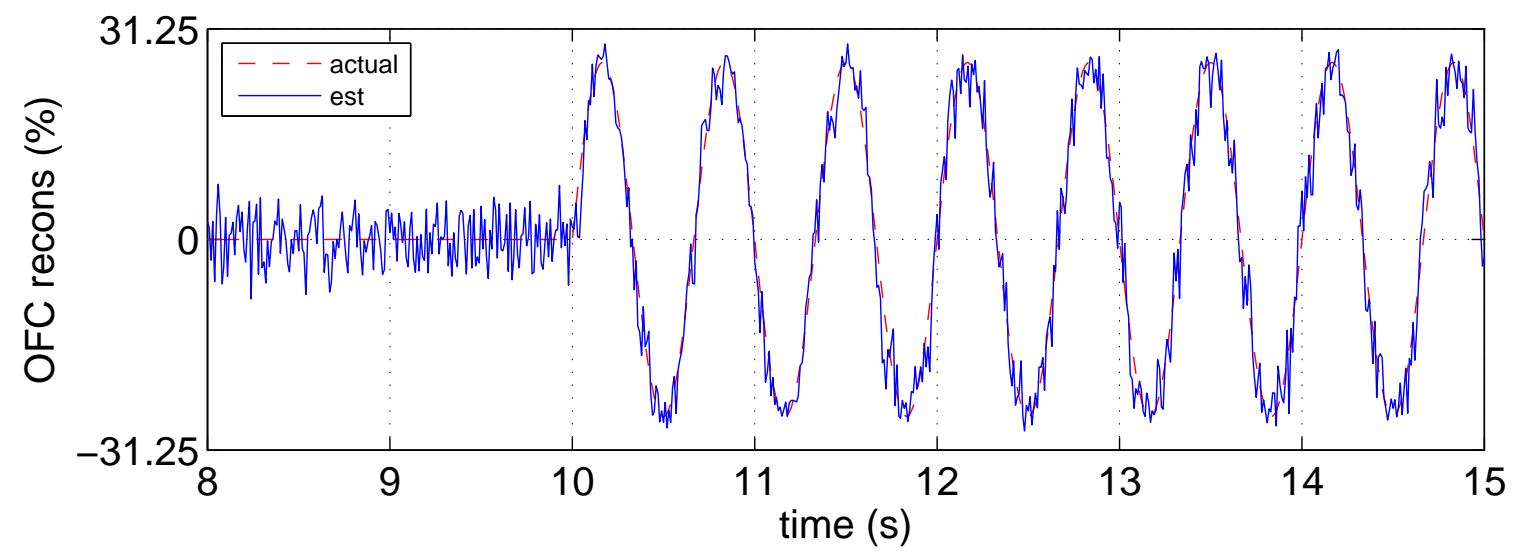

(c) OFC reconstruction

Figure 2: Liquid OFC (medium amplitude, medium frequency) 

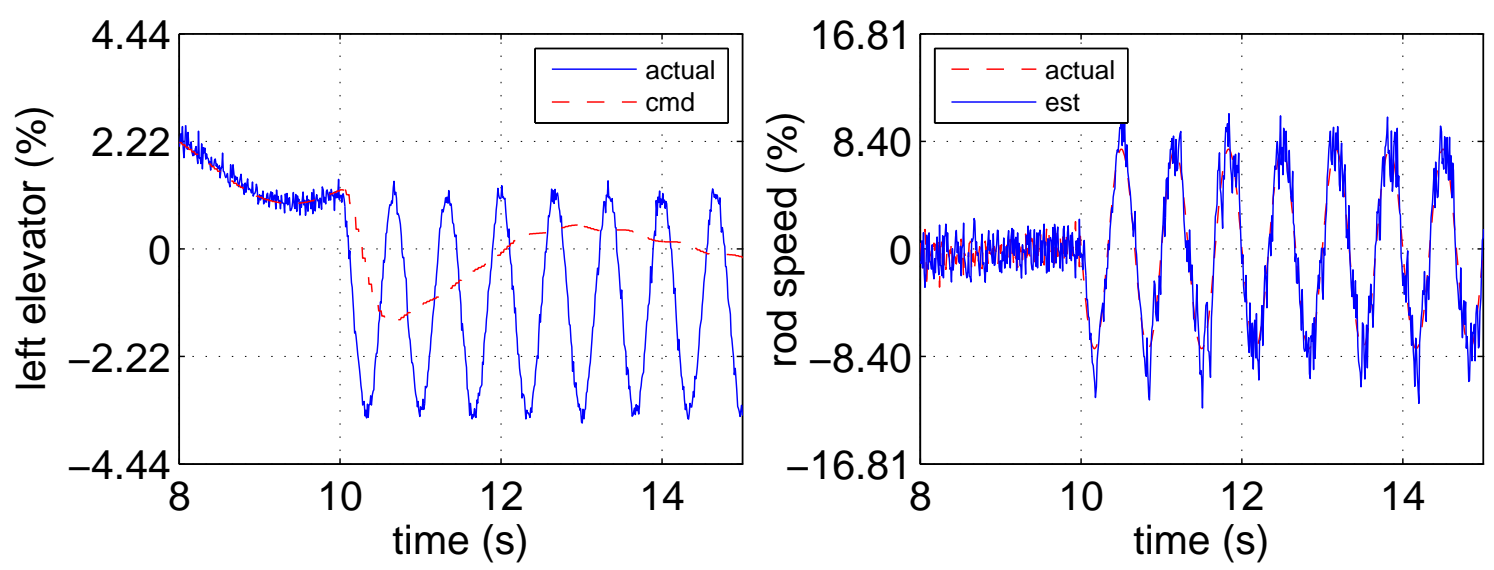

(a) control surface deflection and rod speed
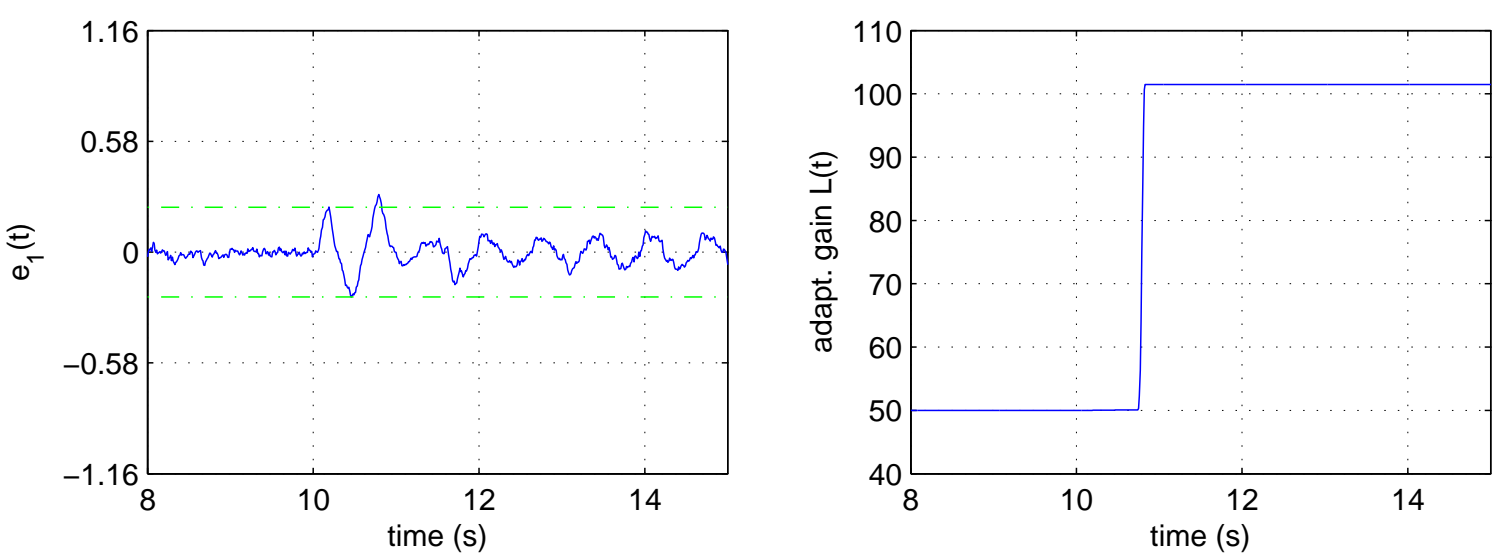

(b) observer performance

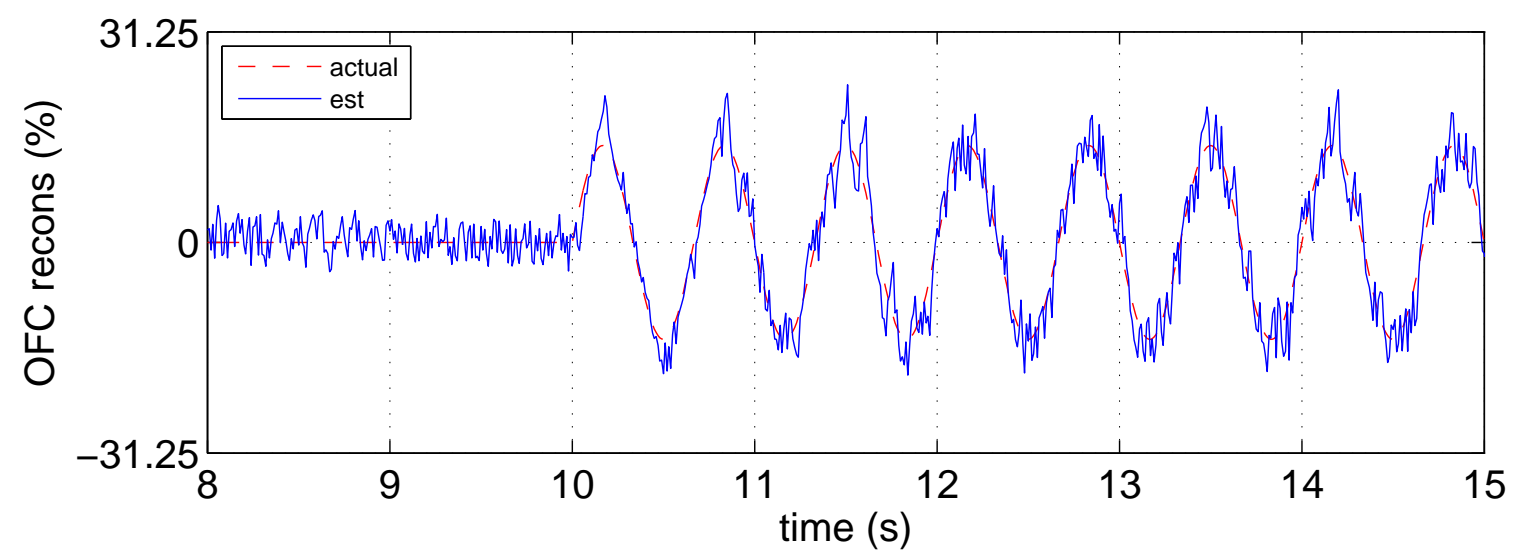

(c) OFC reconstruction

Figure 3: Solid OFC (medium amplitude, medium frequency) 

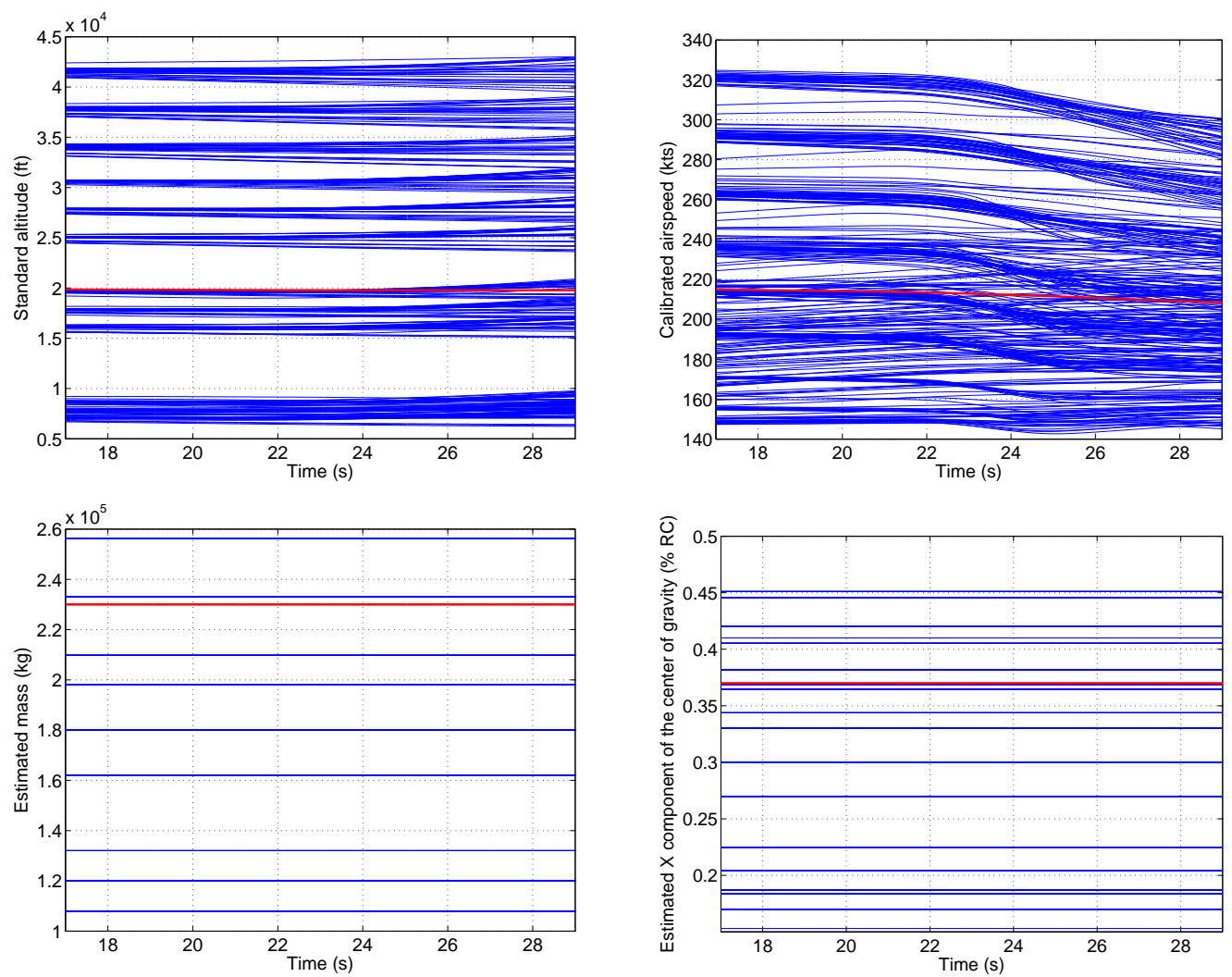

(a) states variations
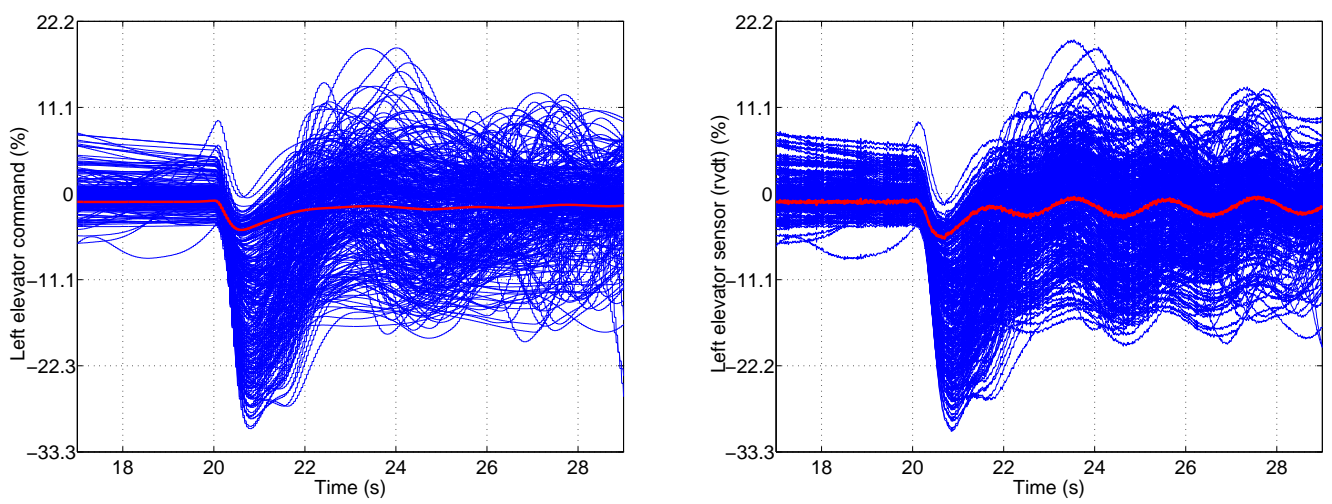

(b) right elevator command and surface deflection
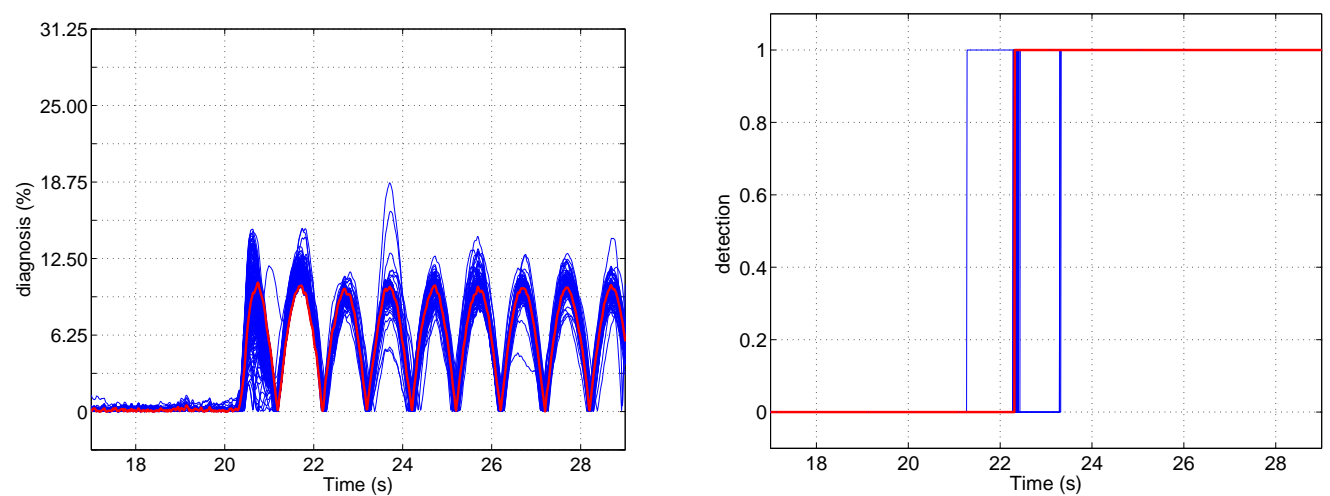

(c) fault reconstruction and detection

Figure 4: Left elevator liquid OFC - parametric run 


\begin{tabular}{|c|c|c|c|c|}
\hline $\begin{array}{c}\text { fault } \\
\text { type }\end{array}$ & amplitude & frequency & $\begin{array}{c}\text { true } \\
\text { detection (\%) }\end{array}$ & $\begin{array}{c}t_{D_{f d d}} \\
\text { max }\end{array}$ \\
\hline Fault free: cruise ADIRS & & N/A & 100 & N/A \\
Fault free: pitch protection & & N/A & 100 & N/A \\
Fault free: yaw angle mode & & N/A & 100 & N/A \\
Fault free: AoA protection & & N/A & 100 & N/A \\
fault free: turn coordination & & N/A & 100 & N/A \\
Fault free: nose up & & N/A & 100 & N/A \\
\hline Solid OFC-A & small & slow & 100 & 0.3683 \\
Solid OFC-B & small & medium & 100 & 0.5000 \\
Solid OFC-C & small & fast & 100 & 0.2800 \\
Solid OFC-D & big & slow & 100 & 0.3433 \\
Solid OFC-E & big & medium & 100 & 0.4900 \\
Solid OFC-F & big & fast & 100 & 0.2333 \\
liquid OFC-A & small & slow & 100 & 0.5017 \\
liquid OFC-B & small & medium & 100 & 0.7850 \\
liquid OFC-C & small & fast & 100 & 0.3033 \\
liquid OFC-D & big & slow & 100 & 0.3333 \\
liquid OFC-E & big & medium & 100 & 0.6250 \\
liquid OFC-F & big & fast & 100 & 0.2800 \\
\hline
\end{tabular}

Table 4: Parametric FES: left elevator OFC

reinforcement reduction translates to a reduction in weight and consequently a reduction in fuel consumption.

The work described in $[31,32,33,34,35,28]$ are examples of other approaches to actuator jam/runaway failure detection within ADDSAFE. The work in [31, 34] uses a mathematical model of the actuator to design the FDD scheme and therefore requires a low order observer scheme. In [35] an LPV representation of the nonlinear actuator model is used as the basis for the observer design. The work in $[32,33]$ consider a more 'global' approach where a typical lateral aircraft model is used as the basis for an observer to generate residuals which are evaluated to detect the presence of an actuator jam or runaway.

In the ADDSAFE benchmark model, the jam and runaway faults are modelled as an additive fault in the output of the actuator servo loop $[16,13,14]$. The idea proposed in this paper is to use the nonlinear local actuator nonlinear model for the observer design and to assume that the output is prone to faults. The output error injection signal associated with the second order sliding mode scheme is used to reconstruct the fault.

\subsection{Modelling of hydraulic actuator for jam/runaway detection}

From (1) and (2), the fault free actuator model can also be written as

$$
\begin{aligned}
\dot{x}(t) & =-K(t) x(t)+K(t) u(t) \\
y(t) & =x(t)
\end{aligned}
$$

where

$$
K(t)=K_{c} K\left(\frac{\Delta_{p_{t o t}}(t)}{\Delta_{p_{\text {ref }}}}\right)^{\frac{1}{2}}
$$

In $(22), x(t)$ is again the rod position and $u(t)$ is the command from the FCC. Importantly for what follows, the scalar $K(t)>0$ for all time [36, 34]. For the jam and runaway failure cases considered in ADDSAFE, the fault is modelled as a perturbation which occurs at the output of the actuator $[14,13,19]$ and therefore (22)-(23) becomes

$$
\begin{aligned}
\dot{x}(t) & =-K(t) x(t)+K(t) u(t) \\
y(t) & =x(t)+f_{o}(t)
\end{aligned}
$$


where the signal $f_{o}(t)$ represents the fault.

\subsection{Sliding Mode Observer}

In (26), the fault appears additively at the output of the actuator model. This is inconvenient from a sliding mode observer perspective. For this reason consider a new state $z_{f}(t) \in \mathbb{R}$ which is the filtered output of $y(t)$ given by

$$
\dot{z}_{f}(t)=-A_{f} z_{f}(t)+A_{f} y(t)
$$

where $A_{f}$ is a positive scalar which represents the bandwidth of the filter in (27). Substituting for $y(t)$ from (26) into (27) and augmenting the plant model in (25) creates a second order system

$$
\begin{aligned}
& \underbrace{\left[\begin{array}{c}
\dot{x}(t) \\
\dot{z}_{f}(t)
\end{array}\right]}_{\dot{x}_{a}(t)}=\underbrace{\left[\begin{array}{cc}
-K(t) & 0 \\
A_{f} & -A_{f}
\end{array}\right]}_{A_{a}(t)} \underbrace{\left[\begin{array}{c}
x(t) \\
z_{f}(t)
\end{array}\right]}_{x_{a}(t)}+\underbrace{\left[\begin{array}{c}
K(t) \\
0
\end{array}\right]}_{B_{a}(t)} u(t)+\underbrace{\left[\begin{array}{c}
0 \\
A_{f}
\end{array}\right]}_{F_{a}} f_{o}(t) \\
& z_{f}(t)=\underbrace{\left[\begin{array}{ll}
0 & 1
\end{array}\right]}_{C_{a}} x_{a}(t)
\end{aligned}
$$

In (28)-(29) the fault now apparently affects the dynamics of the augmented system, but if $z_{f}$ is treated as the output, this signal is a 'pure' fault-free measurement of (part of) the states $x_{a}(t)$. The objective is now to design a sliding mode observer for the system in (28)-(29) to estimate the fault $f_{o}(t)$. For the augmented system (28), the proposed observer has the structure

$$
\begin{aligned}
& \dot{\hat{x}}_{a}(t)=A_{a}(t) \hat{x}_{a}(t)+B_{a}(t) u-G_{l} e_{z}(t)+G_{n} \nu(t) \\
& \hat{z}_{f}(t)=C_{a} \hat{x}_{a}(t)
\end{aligned}
$$

where $e_{z}(t)=\hat{z}_{f}(t)-z_{f}(t)$. The parameters $G_{l}, G_{n} \in \mathbb{R}^{2 \times 1}$ are the observer gains and $\nu(t)$ is the nonlinear term to induce a 2 nd order sliding motion given by

$$
\begin{aligned}
& \nu(t)=-k_{a} \operatorname{sign}\left(e_{z}(t)\right)\left|e_{z}(t)\right|^{1 / 2}+z(t) \\
& \dot{z}(t)=-k_{c} \operatorname{sign}\left(e_{z}(t)\right)-k_{d} e_{z}(t)
\end{aligned}
$$

where the variables $k_{a}, k_{c}, k_{d}$ are design scalars. Consider an (augmented) state error vector $e_{a}(t)=$ $\hat{x}_{a}-x_{a}$, then subtracting (28) from (30) yields

$$
\dot{e}_{a}(t)=A_{a}(t) e_{a}(t)-G_{l} e_{z}(t)+G_{n} \nu(t)-F_{a} f_{o}(t)
$$

The objective is to force $e_{z}(t)=\dot{e}_{z}(t)=0$ in finite time in order to achieve a 2nd order sliding mode. In this paper the observer gains have been chosen as

$$
G_{n}=\left[\begin{array}{l}
0 \\
1
\end{array}\right], G_{l}=\left[\begin{array}{c}
0 \\
-A_{f}+k_{b}
\end{array}\right]
$$

where $k_{b}$ is a positive scalar. Substituting (35) into (34), the error system can be written in augmented form as

$$
\left[\begin{array}{c}
\dot{e}_{x}(t) \\
\dot{e}_{z}(t)
\end{array}\right]=\left[\begin{array}{cc}
-K(t) & 0 \\
A_{f} & -k_{b}
\end{array}\right]\left[\begin{array}{c}
e_{x}(t) \\
e_{z}(t)
\end{array}\right]+\left[\begin{array}{l}
0 \\
1
\end{array}\right] \nu(t)-\left[\begin{array}{c}
0 \\
A_{f}
\end{array}\right] f_{o}(t)
$$

where $\operatorname{Col}\left(e_{x}(t), e_{z}(t)\right)=e_{a}(t)$. From (36) the reduced order sliding motion is given by

$$
\dot{e}_{x}(t)=-K(t) e_{x}
$$

Since $K(t)>0([36,34])$, the reduced order sliding motion dynamics in $(37)$, although time varying, is stable. From the lower equation of (36)

$$
\begin{aligned}
\dot{e}_{z}(t) & =A_{f} e_{x}-k_{b} e_{z}(t)+\nu(t)-A_{f} f_{o}(t) \\
& =-k_{b} e_{z}(t)+\phi(t)+\nu(t)
\end{aligned}
$$


where $\phi(t)=A_{f}\left(e_{x}(t)-f_{o}(t)\right)$. Suppose $e_{x}(t) \in I_{o}$ where $I_{o}$ is a bounded interval of $\mathbb{R}$ containing the origin, then

$$
\begin{aligned}
|\dot{\phi}(t)| & \leq A_{f}\left(\left|\dot{e}_{x}(t)\right|+\left|\dot{f}_{o}(t)\right|\right. \\
& =A_{f}\left(K(t)\left|e_{x}(t)\right|+\left|\dot{f}_{o}(t)\right|\right)
\end{aligned}
$$

where $K(t)>0[36,34]$, and so provided $\dot{f}_{o}(t)$ is bounded $|\dot{\phi}(t)|<\ell$ for some positive scalar. If the gains $k_{a}, k_{b}, k_{c}$ and $k_{d}$ are chosen such that

$$
\begin{aligned}
k_{a} & >2 \sqrt{\ell} \\
k_{b} & >0 \\
k_{c} & >\ell \\
k_{d} & >\frac{k_{b}^{2}\left(k_{a}^{3}+\frac{5}{4} k_{a}^{2}+\frac{5}{2}\left(k_{c}-\ell\right)\right)}{k_{a}\left(k_{c}-\ell\right)}
\end{aligned}
$$

then $e_{z}(t)=\dot{e}_{z}(t)=0$ in finite time [9]. Since during the sliding motion $e_{z}=\dot{e}_{z}=0$, and $e_{x}(t) \rightarrow 0$ since (37) is stable, equation (38) reduces to

$$
\nu(t)=A_{f} f_{o}(t)
$$

From (46) a fault estimate of the form

$$
\hat{f}_{o}(t)=A_{f}^{-1} \nu(t)
$$

can be created which is available online.

The observer described above is parameterized by five scalar parameters $A_{f}$ and $k_{a}, k_{b}, k_{c}$ and $k_{d}$. However $k_{a}, k_{b}, k_{c}$ and $k_{d}$ must satisfy the four inequalities in (42)-(45) and so the actual number of degrees of freedom is less. In fact the gains $k_{a}, k_{b}, k_{c}$ and $k_{d}$ can be parameterized in terms of a single scalar $\ell$ which can be thought of as an upper bound on the rate of change of the fault signal $f_{o}(\mathrm{t})$.

Remark: Note that the structure in (32)-(33) is similar to (10)-(11) except that the gains are fixed. In Section 3 the gains were allowed to adapt - largely to cope with noise on the rod position measurement. In this section the measurement is first filtered according to (27), primarily to create a relative degree one unknown input system formulation, but a bi-product of this is that the pseudooutput $z_{f}(t)$, used to form $e_{z}(t)$, is based on a low pass filtered version of the measurements, which is advantageous from a noise perspective. Consequently there is no necessity to employ (a more complex) adaptive scheme for the gains.

\subsection{Right Elevator Jam/Runaway: Detection Logic}

Simple detection logic monitors the reconstruction signal in (47). Once a certain threshold is exceeded, a fault is assumed to be present and therefore detected.

\subsection{Simulations}

For the elevator jam and runaway fault cases, three levels of simulation results are presented:

1. Single runs: involving no uncertainty to demonstrate the correctness of the implementation.

2. FES parametric runs: with grid parametric uncertainty which are used to tune the FDD design in preparation for the industrial evaluation.

3. FES Monte-Carlo runs: full Monte Carlo test results from the industrial evaluation environment.

In the design which follows, the scalar $A_{f}$ which defines the output filter from (27) has been chosen as $A_{f}=0.5$. The super-twisting gains from (42)-(45) have been chosen as $k_{a}=6.6408, k_{b}=1.0, k_{c}=$ $20, k_{d}=0.3279$.

Remark: Note that specific values of the aircraft parameters are not given here to preserve confidentiality agreements with AIRBUS. Also note that some of the plots in the following figures are expressed in terms of percentage of the admissible range, rather than SI units. 

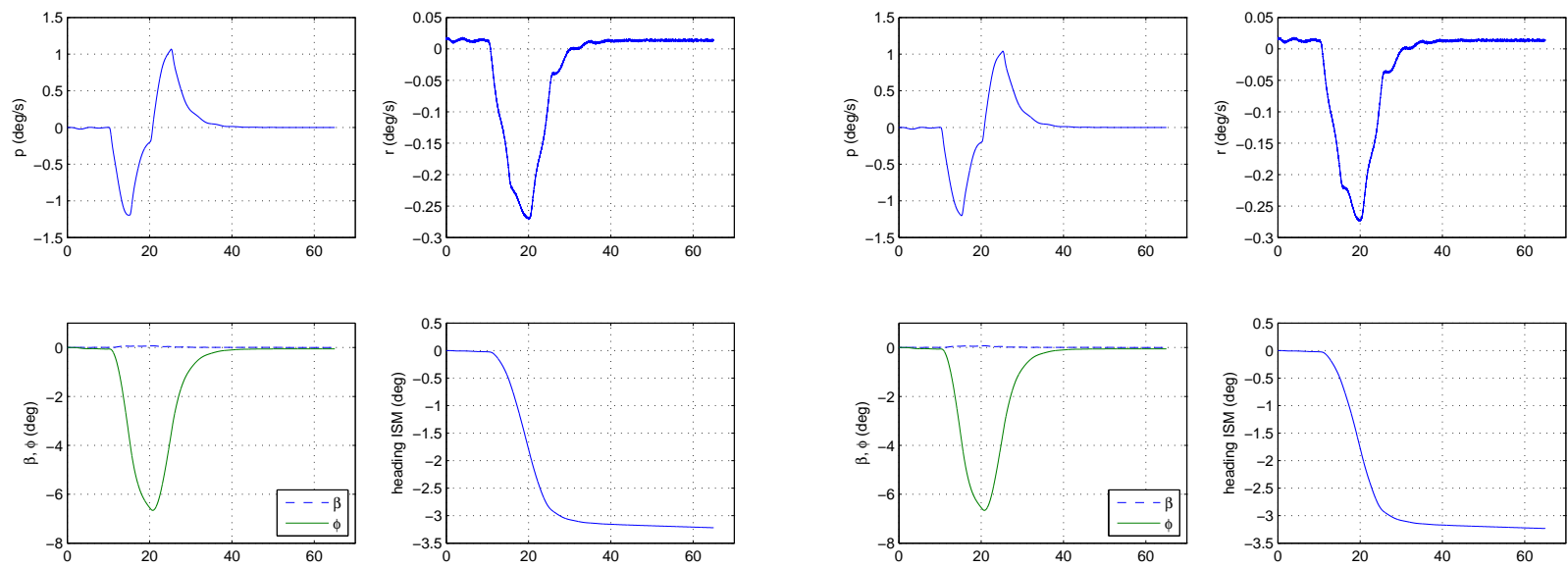

(a) states
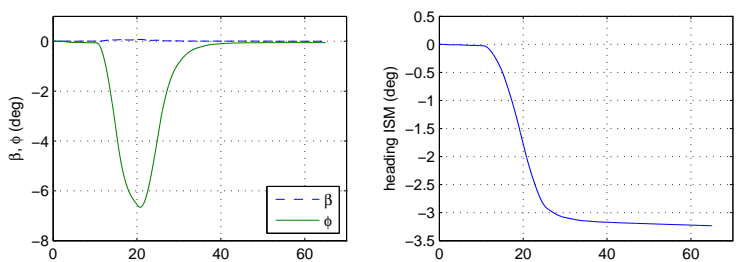

(a) states
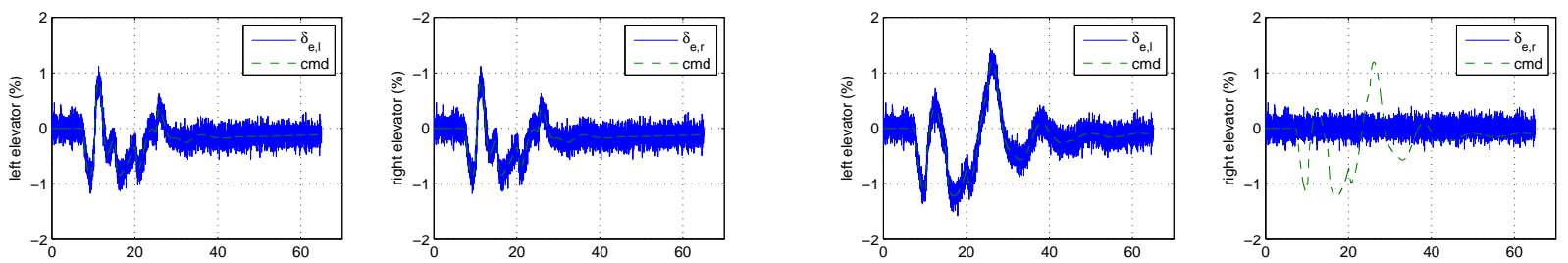

(b) elevator deflections
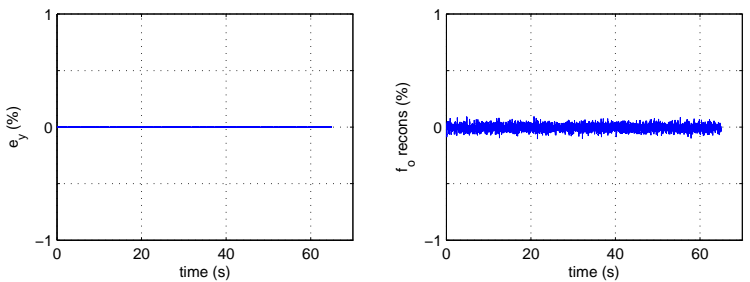

(c) $e_{y}$ and fault estimations

(b) elevator deflections
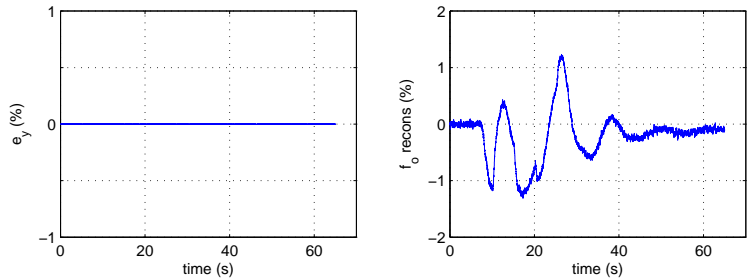

(c) $e_{y}$ and fault estimations

Figure 5: turn coordination: fault free

Figure 6: turn coordination: right elevator jam

\subsection{Single run Simulation Results}

The scheme proposed above has been initially tested on the nonlinear high fidelity ADDSAFE benchmark model and the control surface considered is the right elevator. The simulations are conducted at an altitude of $37000 \mathrm{ft}$, a speed of $267 \mathrm{kts}$, a weight of 185 tonnes and centre of gravity of $28 \%$ MAC. Figures 5-8 show the results of the FDD scheme applied to the right elevator. The results are obtained at a single trim without any parametric and uncertainty variations. Figure 5 shows the fault free performance of the observer while performing a coordinated turn. Figure 5(a) shows the lateral states, and a heading change is clearly seen. The fault free surface deflections can be seen in Figure 5(b), where the elevator deflection follows the demanded position. Finally Figure 5(c) shows that sliding occurs (i.e. $e_{y} \approx 0$ ) and no fault is present (as shown by the estimated fault $\hat{f}_{o} \approx 0$ ). Figure 6 shows the results from a right elevator jam during the coordinated turn manoeuvre. Figure 6(b) shows that the right elevator jams and does not respond to the command signal. Figure 6(c) demonstrates that sliding is being maintained despite the presence of the fault. Figure 6(c) also shows good reconstruction of the fault.

Figure 7 shows the results for a fault free cruise flight. The longitudinal states are shown in Figure $7(\mathrm{a})$, while the surface deflection is shown in Figure 7(b). Figure 7(c) shows no fault is present since the reconstruction signals are close to zero. Figure 8 shows the results of a right elevator runaway during cruise flight. Figure 8(a) shows the effect of the elevator runaway on the longitudinal states. Figure $8(\mathrm{~b})$ shows the effect of a right elevator runaway at $5 \mathrm{sec}$ which does not respond to the commanded signal. Figure 8(c) shows that despite the large magnitude of the fault, sliding is still 

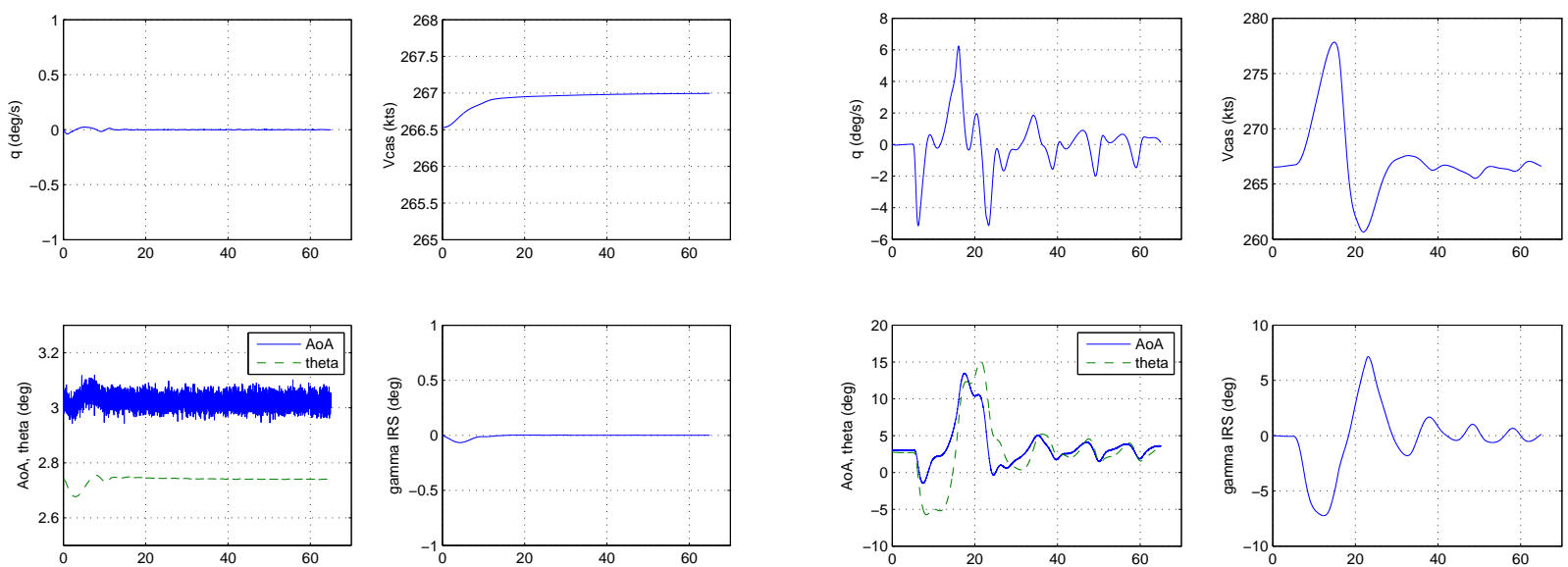

(a) states
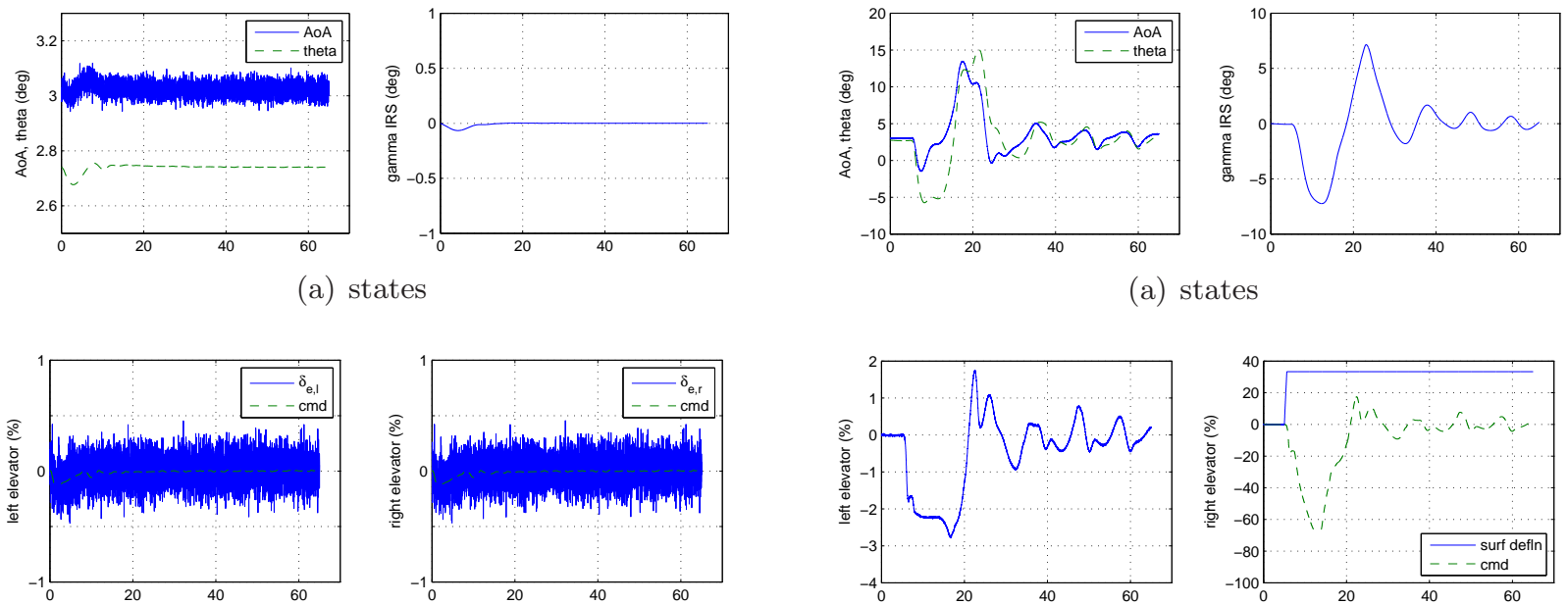

(a) states

(b) elevator deflections
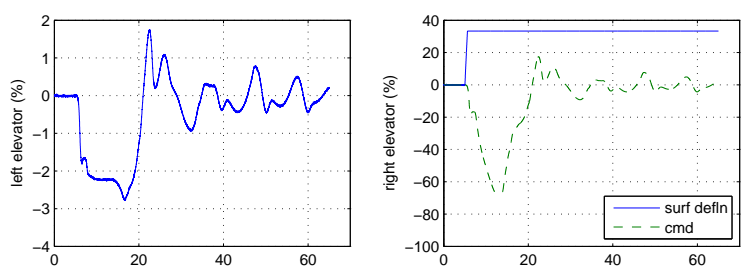

(b) elevator deflections
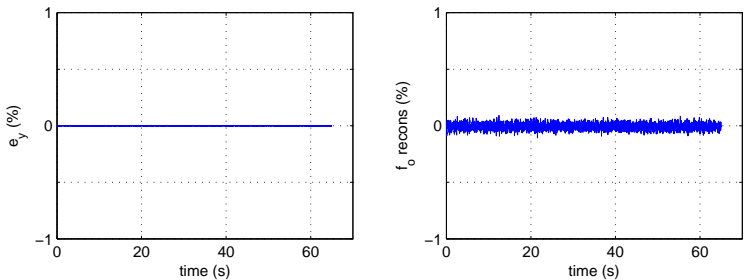

(c) $e_{y}$ and fault estimations
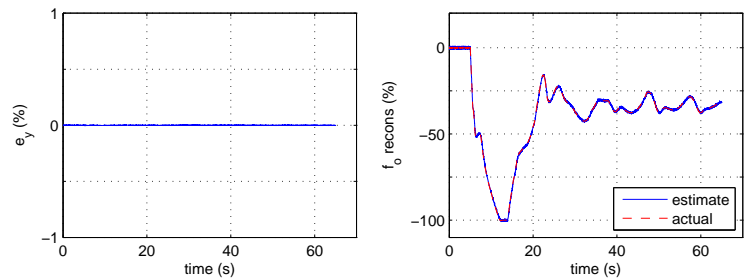

(c) $e_{y}$ and fault estimations

Figure 7: cruise condition: fault free

Figure 8: cruise condition: right elevator runaway

being maintained. Figure 8(c) also shows good fault reconstruction.

\subsection{Parametric Simulation Results}

\subsubsection{Parametric Run Example: Right Elevator runaway}

Figure 9(a) shows the variation of the states associated with the gridding parameters for all the 324 parametric runs under an elevator runaway fault occurring in cruise conditions. Here the runaway is set to $85 \%$ of the maximum operational rate of the actuator. Figure 9(b) shows the command and the actual surface deflections. The actual elevator deflection shows the effect of a runaway which starts at 5 sec. Figure 9(c) shows the output of the FDD scheme. The nonzero fault reconstruction (labelled as 'diagnosis') shows the presence of a fault, indicated by the detection signal once the reconstruction threshold has been exceeded.

\subsubsection{Parametric Run Example: Right Elevator jam}

Figure 10(a) shows the variation of the states associated with the gridding parameters for all 324 parametric runs when the right elevator jams at 0 deg during a coordinated turn manoeuvre. Figure 10(b) shows the command and the effect of the jammed elevator which starts at 8 sec. Figure 10(c) shows the fault reconstruction signals (labelled as 'diagnosis') which indicate the presence of a fault which triggers the detection signal once the reconstruction threshold has been exceeded. 


\subsubsection{Right Elevator Jam/Runaway: FES statistics}

Table 5 shows the statistical performance assessment for the proposed FDD scheme. It shows good detection times (values less than 1) for all the parametric and flight envelope conditions considered. Note that the magnitudes or rates of faults are expressed as percentages of the maximum admissible range. For example $100 \%$ represents the maximum rate of change that the actuator can achieve during normal operation. Also note that the maximum FDD detection times $t_{D_{f d d}} \max$ are normalized values. A value of 1 indicates that the fault is detected at the required detection time, while any value between 0 and 1 indicates a faster than required detection time (which is desirable).

\begin{tabular}{|c|c|c|c|}
\hline $\begin{array}{c}\text { fault } \\
\text { type }\end{array}$ & $\begin{array}{c}\text { runaway rate } \\
\text { or jam amplitude (\%) }\end{array}$ & $\begin{array}{c}\text { true } \\
\text { detection }(\%)\end{array}$ & $\begin{array}{c}t_{D_{f d d}} \\
\max \end{array}$ \\
\hline Fault free: cruise ADIRS & N/A & 100 & N/A \\
Fault free: pitch protection & N/A & 100 & N/A \\
Fault free: yaw angle mode & N/A & 100 & N/A \\
Fault free: AoA protection & N/A & 100 & N/A \\
fault free: turn coordination & N/A & 100 & N/A \\
Fault free: nose up & N/A & 100 & N/A \\
\hline RE runaway 1 & 83.33 & 100 & 0.7273 \\
RE runaway 2 & 166.67 & 100 & 0.6667 \\
\hline RE jam & 0 & 100 & 0.1908 \\
\hline
\end{tabular}

Table 5: Parametric FES: right elevator jam/runaway

\subsection{Monte-Carlo Simulation Results}

The results presented here were obtained from the industrial benchmarking and validation FES supervised by the industrial partners within ADDSAFE. During the evaluation campaign in the industrial FES, the FDD scheme has been converted from SIMULINK blocks into industrially approved 'SAO' blocks provided by AIRBUS (see [14] for further descriptions of the SAO blocks). The SAO blocks represent a graphical symbol library which are different from typical SIMULINK blocks, and represent only a limited set of mathematical operations. The use of the SAO blocks ensures that a particular FDD scheme is compliant with industrial coding standards for subsequent potential implementation. These blocks allow certified software codes to be generated automatically which can be implemented on the flight control computers used by AIRBUS. The process of industrial validation using SAO blocks has been carried out to ensure that the proposed scheme is at a level of complication which is implementable on the actual flight control computer using industrial design tools and constraints. So for example, matrix multiplication, which is normally represented by a single block in SIMULINK, must be broken down to a series of scalar multiplication and addition operations [14].

For the scheme proposed in this section, the design requires only 67 SAO blocks with an executive time of $5.37 \%$ of the maximum allowed allocated processor time for the ADDSAFE project. This represents a very low computational load on the actual flight control computer.

Table 6 shows the statistical results from the parametric runs for the right elevator runaway scenario (for various different runaway rates) and jam failure cases. These results show no false alarms for the fault free case and good detection times - well within the required detection time. As in Table 5, the magnitudes (or rates) of the faults are expressed in terms of a percentage of the maximum admissible range. The worst case FDD detection time $t_{D_{f d d}} \max$ also represents a normalized value. Here it can be seen that for different runaway rates, the worst case FDD detection time is 0.6 which still represents a good detection time - well within the required specifications (even with the runaway rate well above the normal operational value). 

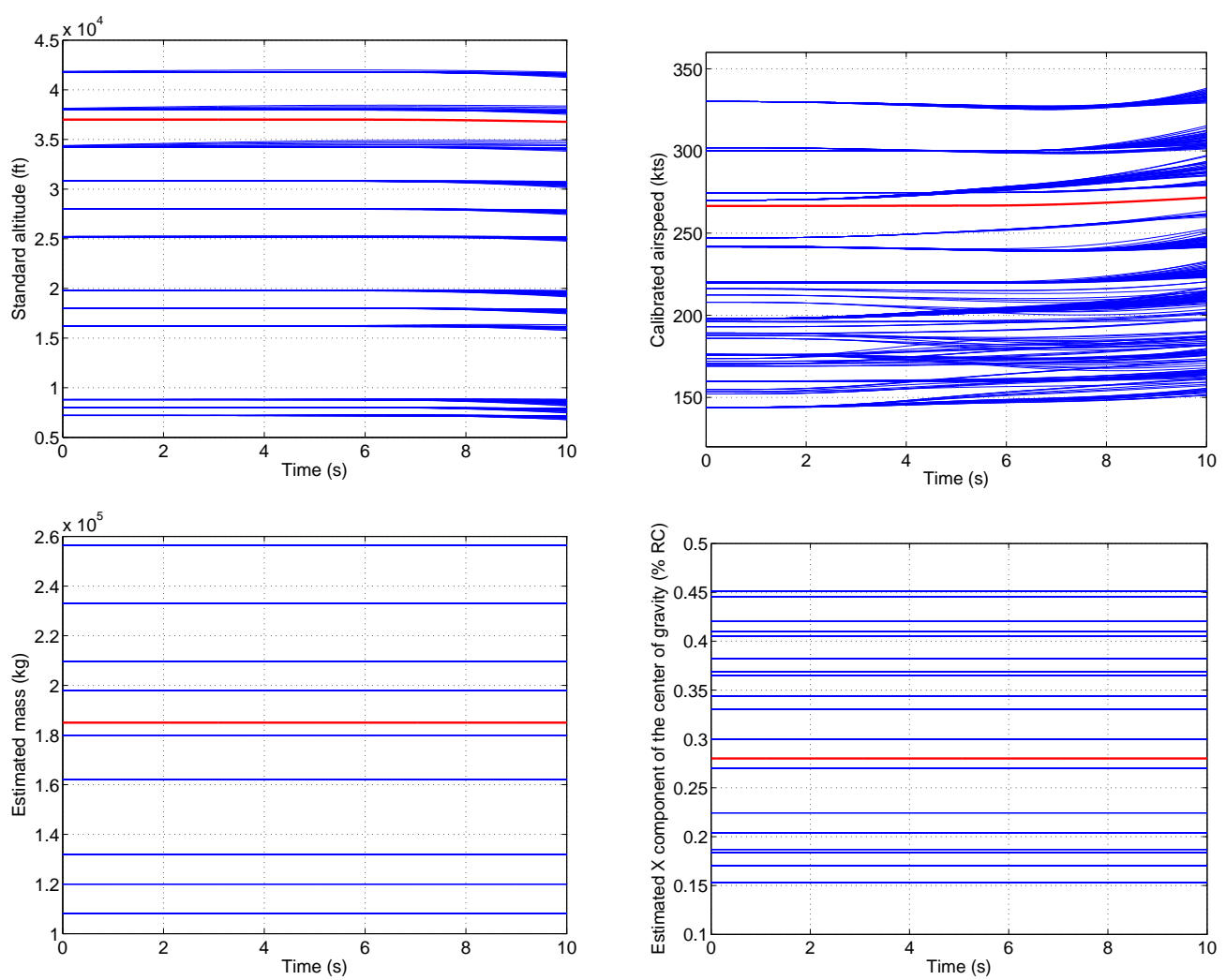

(a) LPV parameter variations
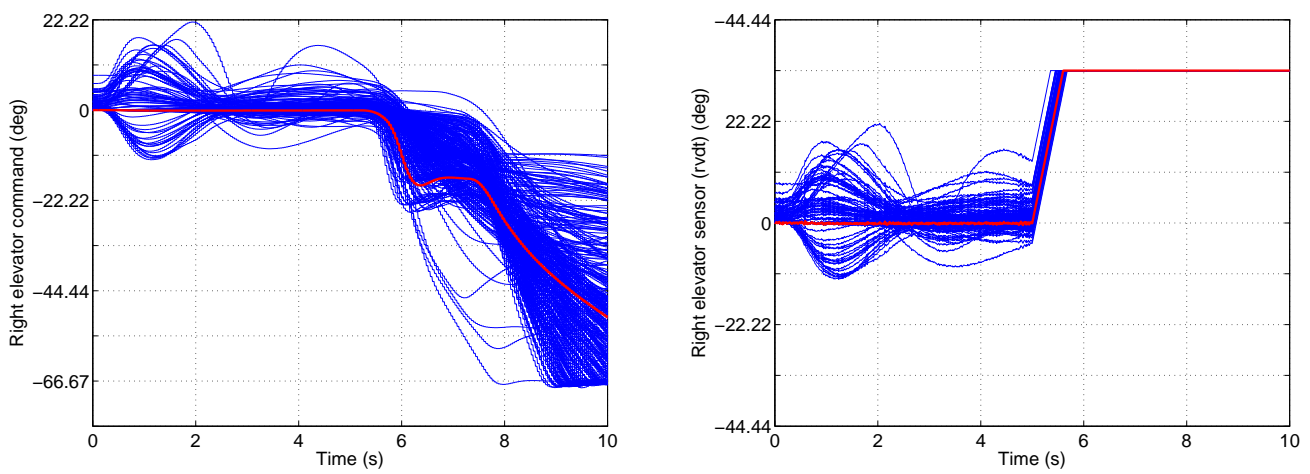

(b) right elevator command and surface deflection
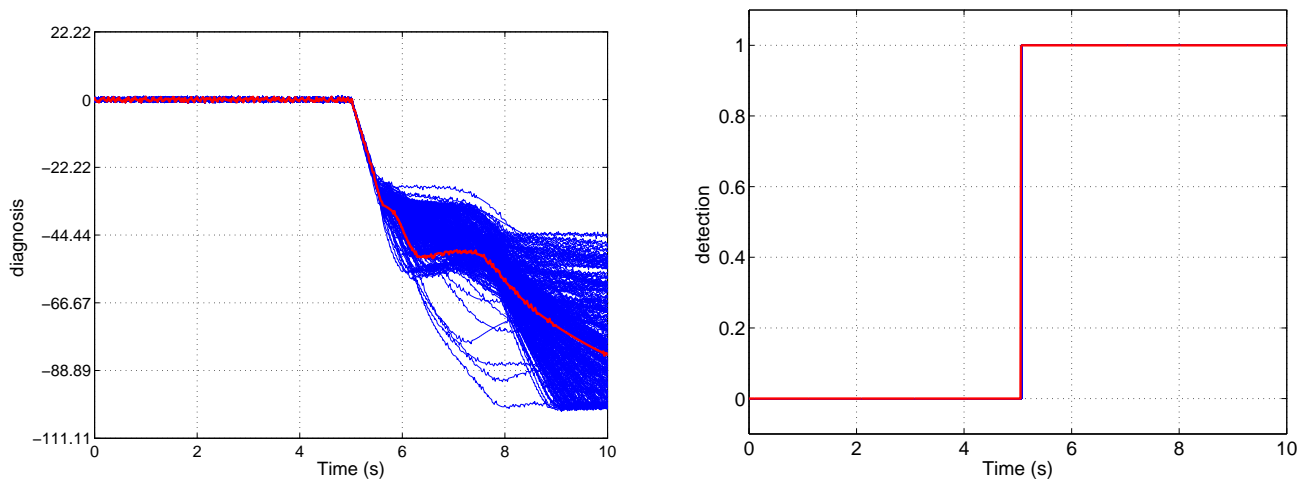

(c) fault reconstruction and detection

Figure 9: Right elevator runaway 

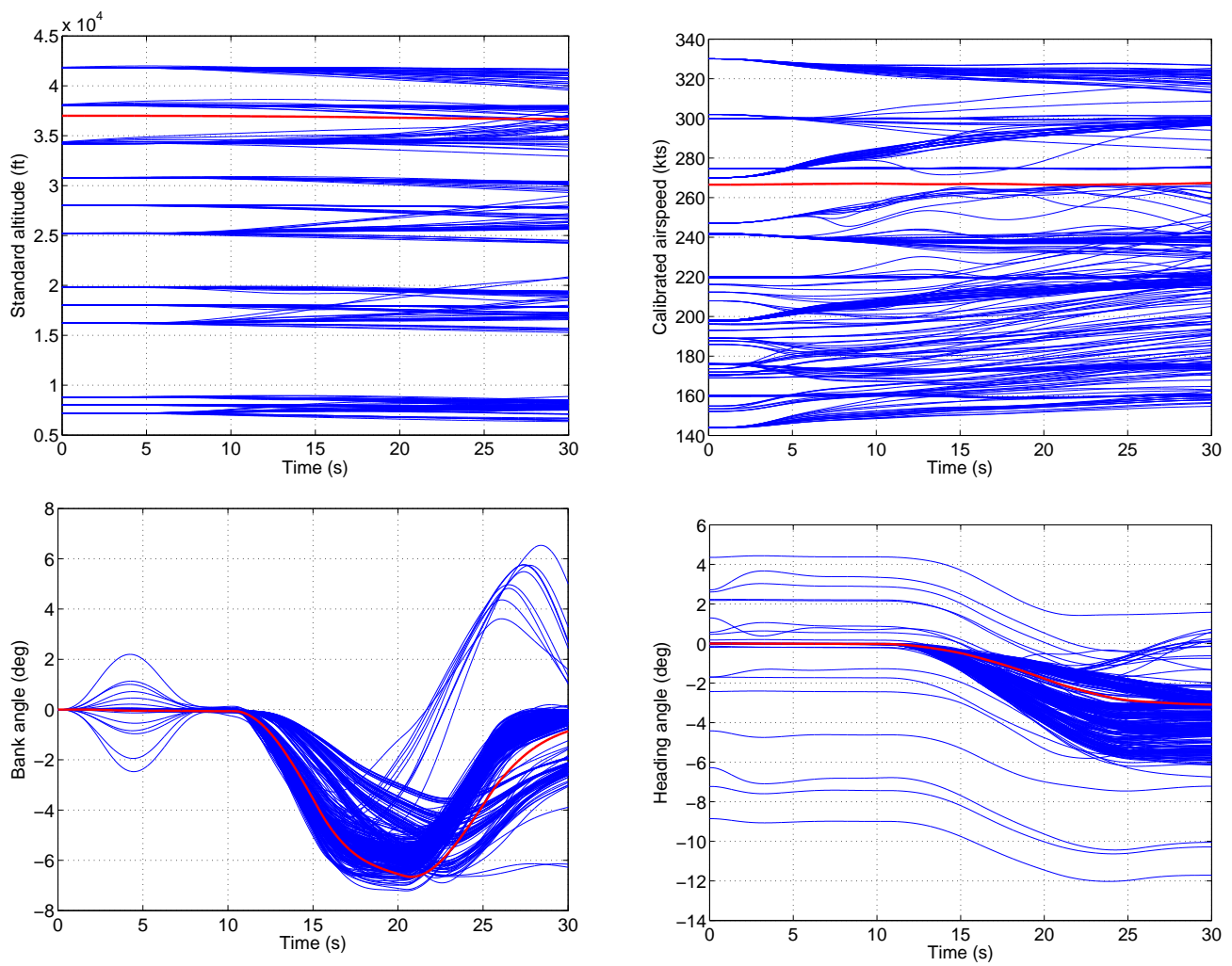

(a) states variations
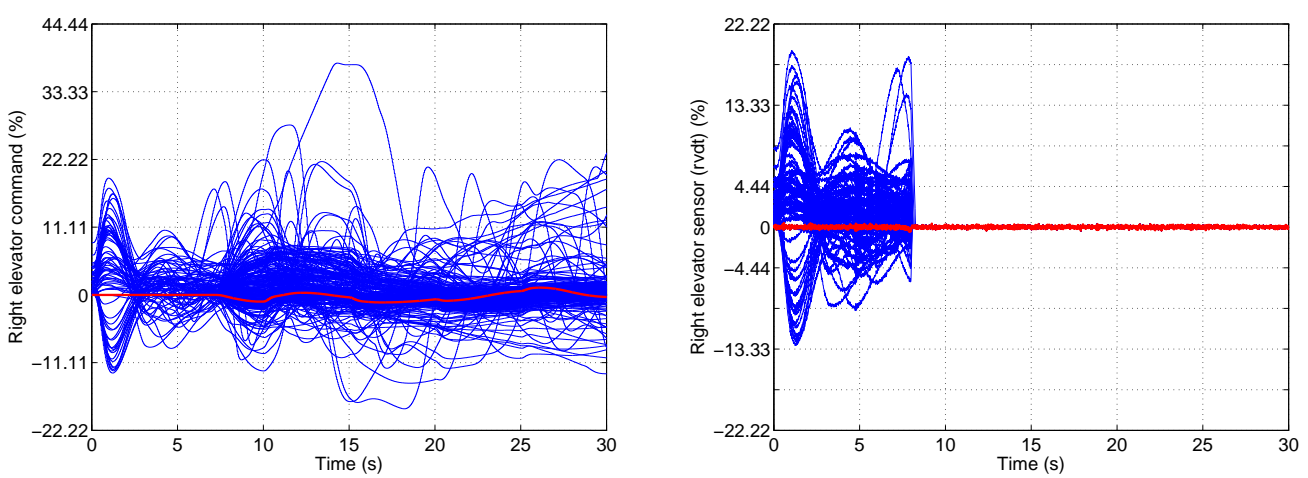

(b) right elevator command and surface deflection
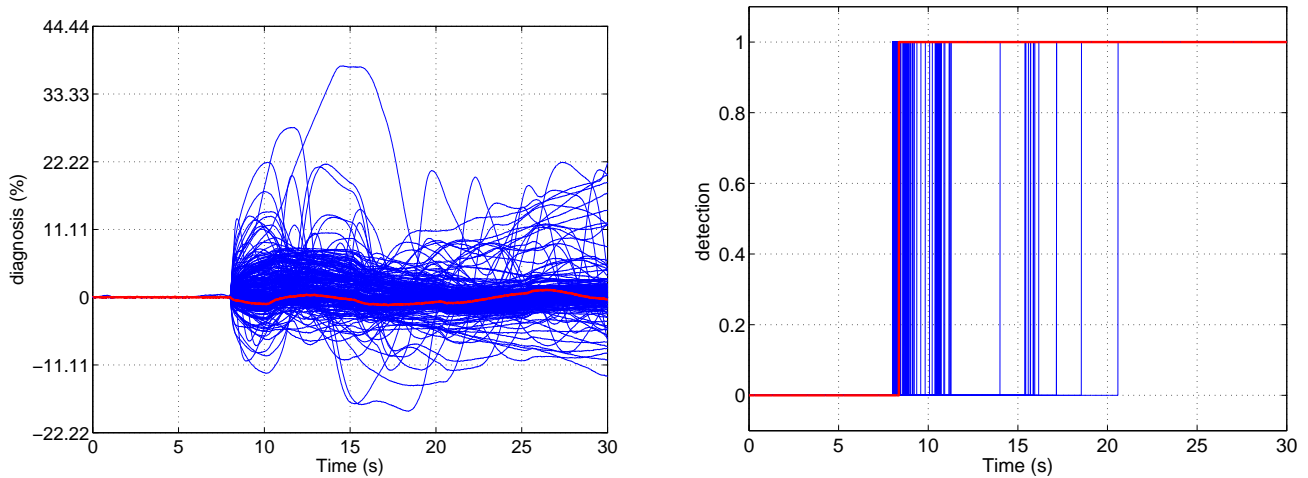

(c) fault reconstruction and detection

Figure 10: Right elevator jam at 0 deg 


\begin{tabular}{|l|r|c|c|}
\hline $\begin{array}{l}\text { fault } \\
\text { type }\end{array}$ & $\begin{array}{r}\text { runaway rate or } \\
\text { jam amplitude (\%) }\end{array}$ & $\begin{array}{c}\text { true } \\
\text { detection }(\%)\end{array}$ & $\begin{array}{c}t_{D_{f d d}} \\
\max \end{array}$ \\
\hline Fault free: cruise ADIRS & $\mathrm{N} / \mathrm{A}$ & 100 & $\mathrm{~N} / \mathrm{A}$ \\
Fault free: pitch protection & $\mathrm{N} / \mathrm{A}$ & 100 & $\mathrm{~N} / \mathrm{A}$ \\
Fault free: yaw angle mode & $\mathrm{N} / \mathrm{A}$ & 100 & $\mathrm{~N} / \mathrm{A}$ \\
Fault free: AoA protection & $\mathrm{N} / \mathrm{A}$ & 100 & $\mathrm{~N} / \mathrm{A}$ \\
fault free: turn coordination & $\mathrm{N} / \mathrm{A}$ & 100 & $\mathrm{~N} / \mathrm{A}$ \\
Fault free: nose up & $\mathrm{N} / \mathrm{A}$ & 100 & $\mathrm{~N} / \mathrm{A}$ \\
\hline RE runaway A & 3.33 & 100 & 0.4080 \\
RE runaway B & 33.33 & 100 & 0.5200 \\
RE runaway C & 66.67 & 100 & 0.4800 \\
RE runaway D & 100.00 & 100 & 0.4802 \\
RE runaway E & 133.33 & 100 & 0.4800 \\
RE runaway F & 166.67 & 100 & 0.6000 \\
\hline RE jam & 0 & 100 & 0.1413 \\
\hline
\end{tabular}

Table 6: Industrial FES: right elevator jam/runaway

\section{Conclusion}

This paper summarizes the design process for two bespoke sliding mode based FDD strategies applied to ADDSAFE benchmark problems. The schemes are quite different, but both are based on a second order sliding mode strategy called super-twisting. Both designs use local actuator models together with local measurements, rather than the global aircraft equations of motion, and consequently the schemes are of low order. Furthermore, in the localized schemes proposed in this paper, the faulty actuator is de-facto isolated which is simpler than a global strategy. For the OFC detection problem, the idea is to use a second order exact differentiator scheme with adaptive gains to estimate the actuator rod speed. This estimate of the rod speed is used to reconstruct any OFC signals that may be present, by mathematical manipulation of the local actuator model. The reconstruction when combined with oscillation counting allows low frequency low amplitude OFCs to be detected quickly.

For high frequency and high amplitude OFCs the fact that adaption of the gain is required to maintain sliding is also exploited to provide faster detection times compared to oscillation counting alone. For the actuator jam/runaway benchmark problem, the design uses a more conventional observer based FDD scheme which provides a reconstruction of the fault. The fault reconstruction is obtained from the equivalent output error injection signals associated with a second order sliding mode structure. The results presented in this paper include an industrial evaluation of the schemes as part of the ADDSAFE project: specifically results obtained from an industrial Functional Engineering Simulator (FES) which uses the full nonlinear ADDSAFE benchmark aircraft model provided by AIRBUS. The results show the evolution of the observer designs from tuning to testing and finally evaluation. Both the proposed FDD scheme designs are subjected to industrial coding constraints and have been evaluated using industrial standard practice. This includes the realization of the schemes using industrially approved SAO blocks provided by AIRBUS, which limit the set of allowable mathematical operations. This ensures that the schemes are compliant with industrial coding standards. Both designs have then been subjected to evaluation by means of an industrial Functional Engineering Simulator to test the performance of the schemes throughout the flight envelope and in the presence of uncertainty. The simulation and evaluation results for both FDD schemes show good robust fault reconstruction for all the tested scenarios. This highlights the potential and efficacy of the proposed schemes.

\section{References}

[1] H. Alwi, C. Edwards, C. P. Tan, Fault Detection and Fault-Tolerant Control Using Sliding Modes, Advances in Industrial Control, Springer-Verlag, 2011. 
[2] C. Edwards, S. K. Spurgeon, Sliding Mode Control: Theory and Applications, Taylor \& Francis, 1998.

[3] V. I. Utkin, Sliding Modes in Control Optimization, Springer-Verlag, Berlin, 1992.

[4] C. P. Tan, C. Edwards, Robust fault reconstruction in uncertain linear systems using multiple sliding mode observers in cascade, IEEE Transactions on Automatic Control 55 (4) (2010) 855 - 867.

[5] L. Fridman, J. Davila, A. Levant, Second-order sliding modes observer for mechanical systems, IEEE Transactions on Automatic Control 50 (2005) 1785-1789.

[6] L. Fridman, J. Davila, A. Levant, High-order sliding mode observation of linear systems with unknown inputs, in: Proceedings of the IFAC World Congress, Seoul, 2008, pp. 4779-4790.

[7] C. Edwards, T. Lombaerts, H. Smaili, (Eds.), Fault Tolerant Flight Control: A Benchmark Challenge, Vol. 399, Springer-Verlag: Lecture Notes in Control and Information Sciences, 2010.

[8] L. Fridman, A. Levant, Higher order sliding modes, in: W. Perruquetti, J. Barbot (Eds.), Sliding Mode Control in Engineering, Marcel Dekker, New York, 2002, pp. 53-96.

[9] J. Moreno, M. Osorio, Strict Lyapunov functions for the super-twisting algorithm, IEEE Transactions on Automatic Control 57 (4) (2012) 1035-1040.

[10] A. Levant, Higher-order sliding modes, differentiation and output-feedback control, International Journal of Control $76(9-10)$ (2003) 924-41.

[11] H. Alwi, C. Edwards, A. Marcos, An Adaptive Sliding Mode Differentiator for Actuator Oscillatory Failure Case Reconstruction, Automatica 49 (2) (2013) 642-651.

[12] P. Goupil, AIRBUS state of the art and practices on FDI and FTC in flight control system, Control Engineering Practice 19 (6) (2011) 524-539.

[13] P. Goupil, A. Marcos, Advanced Diagnosis for Sustainable Flight Guidance and Control: The European ADDSAFE Project, in: SAE AeroTech Congress \& Exhibition, no. doi:10.4271/2011-01-2804, 2011.

[14] P. Goupil, A. Marcos, Industrial benchmarking and evaluation of ADDSAFE FDD designs, in: Proceedings of the IFAC Symposium SAFEPROCESS '12, Mexico City, 2012.

[15] A. Marcos, Advanced fault diagnosis for sustainable flight guidance and control, in: 6th European Aeronautics Days, AERODAYS, Madrid, Spain, 2011.

[16] P. Goupil, AIRBUS benchmark description, Tech. Rep. ADDSAFE D1.1.3, AIRBUS OPERATIONS SAS (2010).

[17] P. Goupil, G. Puyou, A high fidelity AIRBUS benchmark for system fault detection and isolation and flight control law clearance, in: European Conference for AeroSpace Sciences (EUCASS'11), 2011.

[18] V. Fernández, J. M. Ramón, FES software users manual, ADDSAFE Technical Note D1.2.1, DEIMOS (2011).

[19] P. Goupil, FDD problem definition, Report D1.1.2, ADDSAFE (2010).

[20] H. Besch, H. G. Giesseler, J. Schuller, Impact of electronic flight control system (EFCS) failure cases on structural design loads, Agard report 815, loads and requirements for military aircraft (1996).

[21] P. Goupil, Oscillatory failure case detection in the A380 electrical flight control system by analytical redundancy, Control Engineering Practice 18 (9) (2010) 1110-1119.

[22] E. Alcorta-Garcia, A. Zolghadri, P. Goupil, A novel non-linear observer-based approach to oscillatory failure detection, in: European Control Conference, 2009, pp. 1901-1906.

[23] E. Alcorta-Garcia, A. Zolghadri, P. Goupil, L. Lavigne, P. Simon, Nonlinear observer-based OFC detection for A380 aircraft, in: Proceedings of the IFAC Symposium SAFEPROCESS '09, Barcelona, 2009, pp. 47-52.

[24] D. Efimov, A. Zolghadri, J. Cieslak, D. Henry, Input estimation via sliding-mode differentiation for early OFC detection, in: Proceedings of the IFAC Symposium SAFEPROCESS '12, Mexico City, 2012.

[25] D. Efimov, A. Zolghadri, Optimization of fault detection performance for a class of nonlinear systems, International Journal of Robust and Nonlinear Control 22 (17) (2011) 1969 - 1982.

[26] D. Efimov, A. Zolghadri, T. Raissi, Actuator fault detection and compensation under feedback control, Automatica 47 (2011) 1699 - 1705. 
[27] A. Varga, D. Ossmann, LPV-model based identification approach of oscillatory failure cases, in: Proceedings of the IFAC Symposium SAFEPROCESS '12, Mexico City, 2012.

[28] H. Alwi, C. Edwards, Validation of sliding mode observer FDI schemes on the ADDSAFE functional engineering simulator, in: Proceedings of the IFAC Symposium SAFEPROCESS '12, Mexico City, 2012.

[29] A. Levant, Robust exact differentiation via sliding mode technique, Automatica 34 (3) (1998) 379-84.

[30] T. Gonzalez, J. Moreno, L. Fridman, Variable gain super-twisting sliding mode control, IEEE Transactions on Automatic Control 57 (8) (2012) 2100 - 2105.

[31] B. Vanek, Z. Szabó, A. Edelmayer, J. Bokor, Fault detection of electrical flight control system actuators using parameter dependent estimation, in: Proceedings of the IFAC Symposium SAFEPROCESS '12, Mexico City, 2012.

[32] A. Marcos, Application of H-infinity fault diagnosis to ADDSAFE benchmark: the control surface jamming case, in: AIAA Guidance, Navigation and Control Conference and Exhibit, 2011.

[33] L. Chen, R. Patton, P. Goupil, Robust fault estimation and performance evaluation based upon the ADDSAFE benchmark model, in: Proceedings of the IFAC Symposium SAFEPROCESS '12, Mexico City, 2012.

[34] A. Varga, S. Hecker, D. Ossmann, Diagnosis of actuator faults using LPV-gain scheduling techniques, in: AIAA Guidance, Navigation and Control Conference and Exhibit, no. AIAA-2011-6680, 2011.

[35] H. Alwi, C. Edwards, Second order sliding mode observers for the ADDSAFE benchmark problem, in: Proceedings of the IFAC Symposium SAFEPROCESS '12, Mexico City, 2012.

[36] S. Hecker, Nominal and faulty LFT/LPV models, ADDSAFE report D1.3.2-3, DLR (2010). 